\title{
Scanning Near-Field Optical Microscopy on Rough Surfaces: Applications in Chemistry, Biology, and Medicine
}

\author{
Gerd Kaupp \\ Faculty V, Mathematics and Natural Sciences, University of Oldenburg, P.O. Box 2503, 26111 Oldenburg, Germany
}

Received 25 February 2005; Accepted 29 March 2005

\begin{abstract}
Shear-force apertureless scanning near-field optical microscopy (SNOM) with very sharp uncoated tapered waveguides relies on the unexpected enhancement of reflection in the shear-force gap. It is the technique for obtaining chemical (materials) contrast in the optical image of "real world" surfaces that are rough and very rough without topographical artifacts, and it is by far less complicated than other SNOM techniques that can only be used for very flat surfaces. The experimental use of the new photophysical effect is described. The applications of the new technique are manifold. Important mechanistic questions in solid-state chemistry (oxidation, diazotization, photodimerization, surface hydration, hydrolysis) are answered with respect to simultaneous AFM (atomic force microscopy) and detailed crystal packing. Prehistoric petrified bacteria and concomitant pyrite inclusions are also investigated with local RAMAN SNOM. Polymer beads and unstained biological objects (rabbit heart, shrimp eye) allow for nanoscopic analysis of cell organelles. Similarly, human teeth and a cancerous tissue are analyzed. Bladder cancer tissue is clearly differentiated from healthy tissue without staining and this opens a new highly promising diagnostic tool for precancer diagnosis. Industrial applications are demonstrated at the corrosion behavior of dental alloys (withdrawal of a widely used alloy, harmless substitutes), improvement of paper glazing, behavior of blood bags upon storage, quality assessment of metal particle preparations for surface enhanced RAMAN spectroscopy, and determination of diffusion coefficient and light fastness in textile fiber dyeing. The latter applications include fluorescence SNOM. Local fluorescence SNOM is also used in the study of partly aggregating dye nanoparticles within resin/varnish preparations. Unexpected new insights are obtained in all of the various fields that cannot be obtained by other techniques.
\end{abstract}

Copyright (C) 2006 Gerd Kaupp. This is an open access article distributed under the Creative Commons Attribution License, which permits unrestricted use, distribution, and reproduction in any medium, provided the original work is properly cited.

\section{INTRODUCTION}

While most scanning near-field optical microscopy techniques (SNOM) [other acronyms are NSOM (near-field scanning optical microscopy), PSTM (photon scanning tunneling microscopy), and STOM (scanning tunneling optical microscopy)] are only suitable for very flat surfaces, an apertureless procedure in constant shear-force distance [1] is able to achieve artifact-free SNOM contrast on (very) rough surfaces. The slopes may reach angles up to $70^{\circ}-80^{\circ}$ and the achievable resolution is $10 \mathrm{~nm}$. Unlike aperture SNOM [2] and apertureless scattering SNOM in tapping mode [3], only this apertureless technique is adapted to "real world" transparent and opaque samples. Our basic new and unexpected physical effect, which allows for the very good resolution despite an illuminated area of about $1 \mu \mathrm{m}$ diameter, is the strongly enhanced reflection back into the very sharp fiber at shear-force distance $[4,5]$. An illuminated tip with very sharp apex (end radius $10-20 \mathrm{~nm}$ ) must be used. Blunt and broken tips do not provide the enhanced reflection back to the fiber in shear-force distance. These provide a series of possible artifacts that are easily recognized by the drop in recorded light intensity and by some characteristic features [6] that are also apparent in many published images from the other SNOM techniques. The optical contrast is a chemical contrast or materials contrast. It derives from different shearforce response and all other physical effects that affect the reflectivity, which are more diverse than in optical (including coaxial) microscopy. We describe here scientific, medicinal, and industrial applications of the unprecedented tool.

\section{EXPERIMENTAL}

The equipment has been amply described before $[1,7]$. Quartz-glass waveguides are pulled as sharp as possible (end radius $<20 \mathrm{~nm}$ ), laser light is coupled in and the tip horizontally dithered at constant preselected shear-force damping. The strongly enhanced reflected light is collected in the shear-force gap by the fiber and coupled out for detection. Crossed polarizers remove unwanted stray light. The 


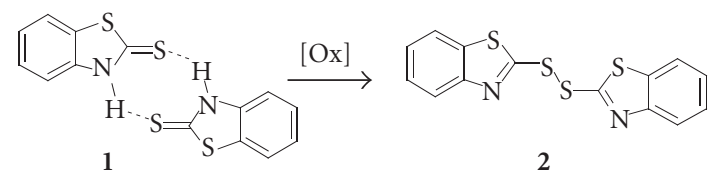

SCHEME 1
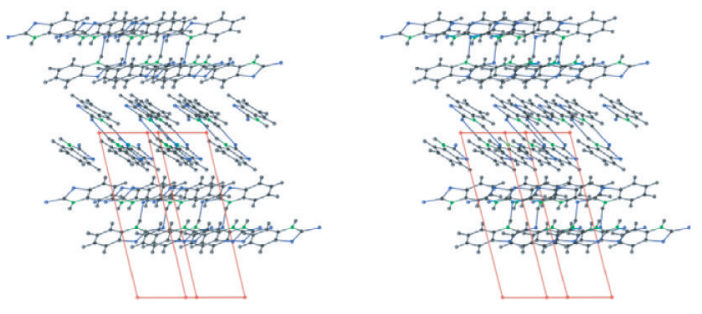

FIGURE 1: Stereoscopic view of the molecular packing of 2-mercaptobenzothiazole (1) with (001) on top showing the flat double layers internally hydrogen-bridged and thus with all functional groups shielded from chemical attack under (001) on the molecular scale.

shear-force feedback (as detected by a second laser) provides the simultaneous AFM topography without any crosstalk. A commercial instrument is used (RASTERSCOPE SNOM 4000 of Danish Microengineering) and the enhancement factor (> 2-50) monitored in every measurement in order to guarantee proper, artifact-free conditions. Natural untreated surfaces are scanned. This includes crystals, polymer beads, (cryo-)microtome cuts that tend to be very rough, and nanoparticle preparations.

\section{APPLICATION OF SNOM IN SOLID-STATE CHEMISTRY}

The chemical contrast of SNOM is an invaluable tool for the evaluation of important mechanistic questions in solid-state chemistry. These questions are very diverse and must be elucidated in the nanoscopic range. A large number of unexpected though environmentally benign sustainable gas-solid reactions exist and have been mechanistically investigated with AFM [8]. The close correlation with the crystal packing and the face selectivity endows them with credence, as they require far-reaching molecular migrations within the crystal, which were previously denied in textbooks claiming the opposite by following Schmidt's topochemistry hypothesis [9]. We present here some typical examples, which support the AFM interpretation of far-reaching migrations within the crystal by the new photophysical tool, the SNOM for rough surfaces.

\subsection{Mercaptobenzothiazole reactions.}

Crystals of mercaptobenzothiazole (1) in the thione tautomeric form $\left(\mathrm{P} 2_{1} / \mathrm{c}\right)$ are remarkably stable towards autoxi-

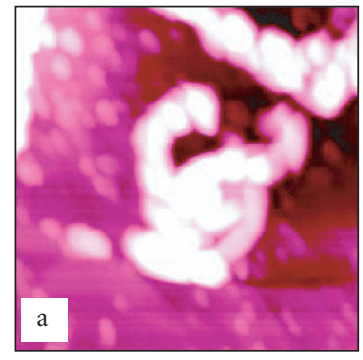

(a)

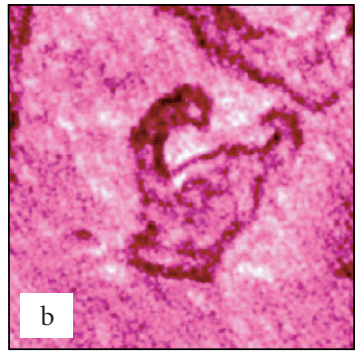

(b)
Figure 2: Simultaneous AFM (a) $(11 \mu \mathrm{m}, \mathrm{Z}$ range $500 \mathrm{~nm})$ and SNOM (b) of a partly autoxidized (001)-face of 2-mercaptobenzothiazole (1) showing islands and negative chemical contrast precisely at the island sites of $\mathbf{2}$.

dation in air (Scheme 1). They provide thin plates with most prominent (001)-faces [10].

AFM measurements on partly autoxidized surfaces exhibit flat areas and eventually isolated islands. This observation can be related to the crystal packing, which exhibits single layers parallel to the surface that are connected in the pairs of double layers by hydrogen bonds. The hydrogen bonds connect two molecular entities to the essentially planar eightring dimer 1 while forcing all reactive groups to the interior of the double layers (Figure 1). Therefore, autoxidation should be impeded and it is concluded that the oxidation product 2 starts to form at some nucleation sites and that the reaction spreads from there. Final submicroscopic proof for this mechanism is provided by SNOM.

Figure 2 shows both the topography of such islands (a) and the dark chemical contrast (b) at the sites of the islands. This proves that oxidized material 2 is only at the islands [10] in the necessary clearcut way.

Similarly, the islands produced by oxidation of 1 with gaseous $\mathrm{NO}_{2}$ or solid 2,2,6,6-tetramethylpiperidinylnitrosonium nitrate [11] or by salt formation (with $\mathrm{CH}_{3} \mathrm{NH}_{2}$ ) on the (001)-face can be elucidated with this new photophysical technique providing similar results: reaction sites are the submicroscopic islands.

\subsection{Anthracene autoxidation.}

Self-protection against autoxidation at the molecular level is observed with anthracene (3). It crystallizes in scales with overwhelming (001)-faces, where the molecules stand on their short side and hide the reactive 9,10-positions ( $>6$ months stable in air with AFM precision). The more elusive prism crystals are also protected: only the reactive 9-positions (but not also the 10-positions) are at the flat (110)-surface (Figure 3), and the crystal is protected against autoxidation except at crystal faults such as the slopes of craters [11].

Prism crystals of anthracene (3) tend to exhibit craters of up to $400 \mathrm{~nm}$ depths at the (110)-face. In that circumstance, both reactive centers are accessible for oxygen attack, 


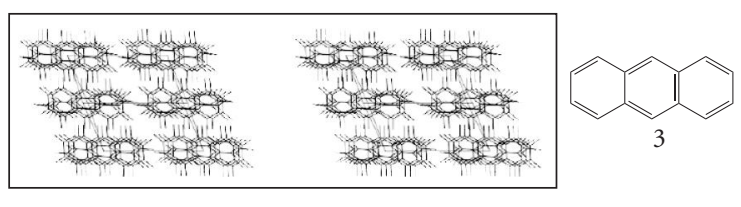

FIgURE 3: Stereoscopic view of the molecular packing of anthracene 3 with (110) on top, showing that both the 9- and 10-positions are only available from the front (or the back); the left side is unreactive (001).

2

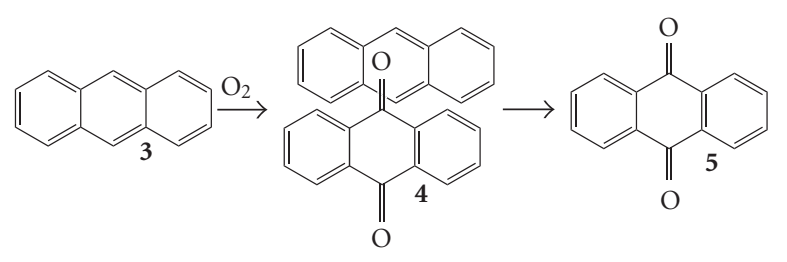

SCHEMe 2

and oxidation to produce 9-, 10-anthraquinone (5) occurs. Neither anthracene (3) nor anthraquinone (5) crystals absorb at $488 \mathrm{~nm}$; however, an intermediate product, the charge transfer sandwich complex between both of them (4) does so, as shown by the pure independently synthesized brown complex (spectrum in [11]).

Only SNOM can reveal the complicated situation at the crystal faults on (110) of anthracene prism crystals (3) that were preexposed to air and started autoxidation there. The AFM topography in Figure 4(a) does not provide chemical information even though the rims on top of the slopes and minute crystallites in the depths point to some reaction with ambient oxygen. However, SNOM proves that autoxidation has started at the slopes and at the rims by providing contrast of $\mathbf{3}$ (normal = plane), $\mathbf{4}($ dark $=$ depth $)$, and $\mathbf{5}($ bright $=$ peak $)$ in Figure 4(b). Clearly, no autoxidation is discernable at the flat sites of the surface (3), 4 is located at the smooth slopes, and $\mathbf{5}$ at the crystallites (rim and depth). Further images are reported in [4].

\subsection{Sulfanilic acid diazotization.}

The performance of shear-force apertureless SNOM might be demonstrated with the gas-solid diazotization of sulfanilic acid monohydrate (6) to give the corresponding diazonium salt 7 by the action of gaseous $\mathrm{NO}_{2}$ (Scheme 3 ) $[10,12,13]$.

The gas-solid diazotization of sulfanilic acid monohydrate with gaseous $\mathrm{NO}_{2}$ comes to a stop on its natural (010)-face, whereas an attack from the sides of the hydrogen bonded infinite ribbons occurs. This is clearly seen in the packing diagram (Figure 5). It appears impossible to have the molecules migrate up over the (010)-surface on top, because the hydrogen bonds keep the molecular strings in their position preventing access to the second layer and so on. Conversely, all layers are accessible from the sides of the crystal.
The conclusion from the crystal packing at the molecular level must and can be secured with SNOM at steps or slopes on the (010)-surface. Figure 6 gives an example. The step site had been searched on the surface by AFM and did not give a SNOM contrast before the application of a small amount of gaseous $\mathrm{NO}_{2}$ indicating previous chemical uniformity. Furthermore, the AFM image did not observably change at the chosen scale after the partial diazotization. The bright contrast (peaks in Figure 6(b)) is only seen at the slopes of Figure 6(a). Thus, the (010)-surface is indeed only covered with a half layer of diazonium salt in the flat parts; whereas more profound diazotization occurs at the slope sites from where the diazonium salts give rise to bright reflection. Longer-term diazotization leads to complete reaction of the whole crystal without melting and without producing wastes.

\subsection{Photodimerization of $\alpha$-cinnamic acid.}

A controversy in solid-state photochemistry required scrutiny by AFM that had to be complemented by SNOM on very rough surface. Thus, $\alpha$-cinnamic acid (8), the standard of Schmidt's topochemistry (claiming absence of molecular migrations [9]), gives submicroscopic features by anisotropic long-range molecular migrations upon irradiation when the dimer $\alpha$-truxillic acid (9) is formed (Scheme 4).

The features correlate strictly with the crystal packing of $\alpha$-cinnamic acid and they therefore constitute definite prove. However, the importance of the topic requires additional proof by independent SNOM. In no stage of the reaction could a SNOM contrast be obtained, therefore the photoreaction proceeds everywhere in the region of light penetration (down to 1300 layers with $365 \mathrm{~nm}$ light or through the whole crystal at "tail irradiation" with extremely low absorbed filtered daylight intensity at an extremely slow partial transformation during 6 months) [14] and the features do not differ in composition at different sites or from flat parts. As the photodimer 9 (without $\mathrm{C}=\mathrm{C}$ double bonds) should reflect very differently from the cinnamic acid (8) (with a $\mathrm{C}=\mathrm{C}$ double bond) any possibility for a cluster-by-clustertype photoreaction before crystal disintegration is additionally excluded by the absence of chemical contrast. No contrast can be seen at and around low and $\mu \mathrm{m}$ high features that grow out of the bulk above the initially flat (010)-surface by molecular migrations over very long distances. The result of Figure 7 (as well as numerous similar measurements at earlier stages of the photolysis under varied conditions) shows that no chemical contrast is discernible whatsoever even at very high features. The highly reliable performance of the applied SNOM is indicated by the very minor steepness contrast at the edges of the huge $(>1 \mu \mathrm{m})$ and steep $\left(45^{\circ}-55^{\circ}\right)$ feature sites in (b). There is no trace of a chemical contrast; the rough surface is thus chemically uniform in final contradiction to the topochemistry claim.

\subsection{Hydrolysis of phthalimide.}

While apertureless shear-force SNOM on rough surfaces is able to help in the development of sustainable and waste-free 


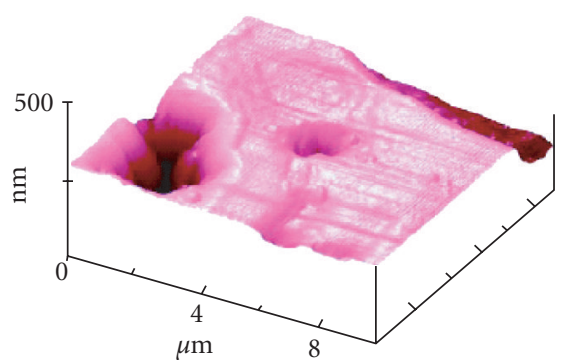

(a)

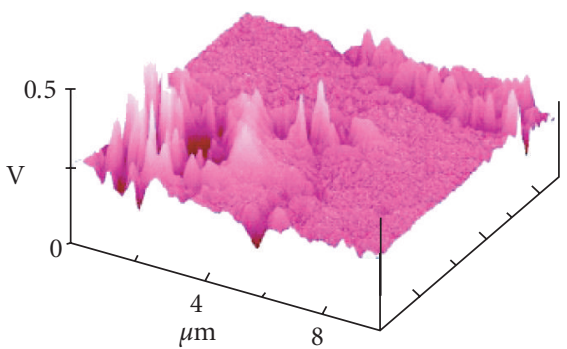

(b)

FIgURE 4: Simultaneous AFM (a) and SNOM (b) on a (110)-face of anthracene (3) (P2 1 /a) with some faults that were preexposed to ambient air; the slopes rarely exceed $20^{\circ}$ (but $30^{\circ}$ is also present); the SNOM image (b) indicates three types of contrast: normal at the flat sites, peak at the rims and depths, and down at the slopes due to different local advance of reaction.

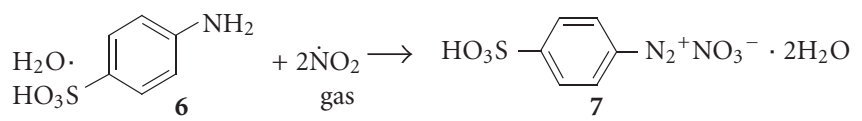

Scheme 3

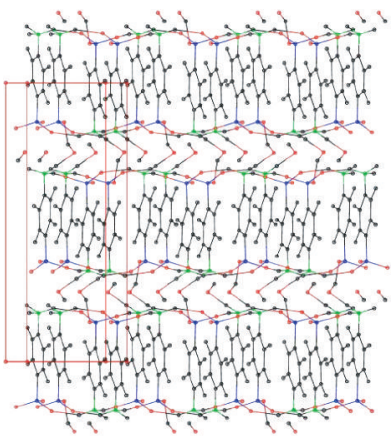

(a)

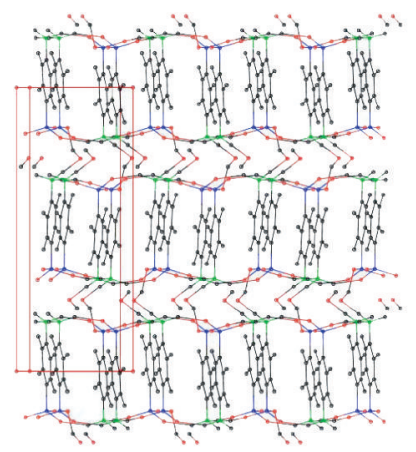

(b)
FIGURE 5: Stereoscopic view of the molecular packing and hydrogen bonding of sulfanilic acid monohydrate (6) along (100) (but rotated around $y$ by $10^{\circ}$ for a better view) with (010) on top showing hydrogen-bridged strings of molecules that can only be chemically attacked from the sides; the (010)-surface will have all accessible amino groups (any other molecule) diazotized.

reactions, it is also able to differentiate between different consecutive chemical reactions. For example, phthalimide (10), a heterocycle with three polar groups can both hydrate the surface and react chemically to give phthalic acid monoamide (Scheme 5). It is a favorable example for the demonstration of single molecular layer effects as the crystal packing on the most prominent (001)-face of $\mathbf{1 0}$ has the functional groups hidden under the hydrophobic benzene rings (Figure 8). However, surface hydration is not excluded, as one of the oxygen atoms per molecule is not efficiently shielded (Figure 8(b)). A double-layer situation as on (001) in Figures 8(a), (b) should be unreactive. Therefore, an island mechanism can be expected at best on that face.

The AFM analysis reveals a very flat initial (001)-surface with molecular steps (bilayers) and some peaks with heights of 2.3-3.3 nm (Figure 9(a)) that persist in moist air. The surface hydration in wet air is the only process within 2 days: a uniform change at the $10 \mathrm{~nm}$ ranges is obtained (Figure 9(b)). Numerous bilayer steps and deeper ditches align along the long crystal axis (010) and are most likely hydrated at least at the molecular steps and beyond. No SNOM contrast was obtained at that stage. More extended exposure to moisture (weeks) or exposure to nearly saturated water vapor for $16 \mathrm{~h}$ produces islands of considerable height (up to $180 \mathrm{~nm}$ ) while the direction of preference is retained as shown in Figure 9(c) (the ditch is now ca. $10 \mathrm{~nm}$ deep). The larger isolated islands are the result of a chemical reaction with long-range molecular migration. Most likely is hydrolytic ring opening to give phthalic acid monoamide only at the islands?

In view of the minute quantities of material, this conclusion is subject to SNOM scrutiny, which is given in Figure 10. The simultaneous AFM and SNOM (at a different site of the above sample) disclose a strong chemical contrast, which is dark in this case and very different from the unreacted hydrated flat plane, in support of the localized chemical reaction. The tip had to go over slopes that never reached $40^{\circ}$ except for one region near the top of the island feature. The reliability of the measurement is demonstrated by the precise local correspondence with the topographic image. The broad and very minor positive features do not seem to be part of the chemical contrast. Similar dark chemical contrast in a more advanced state of hydrolysis has been found with the SNOM applying $633 \mathrm{~nm}$ light [15]. 


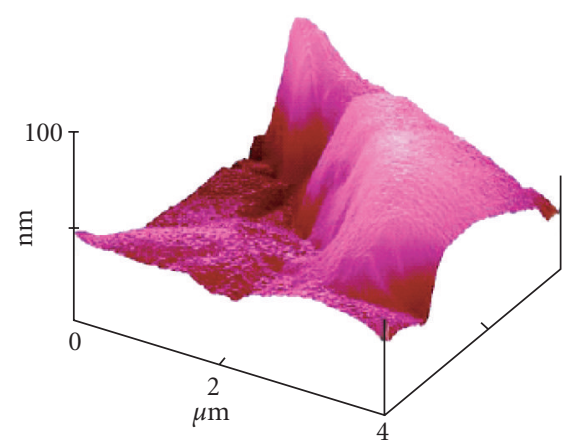

(a)

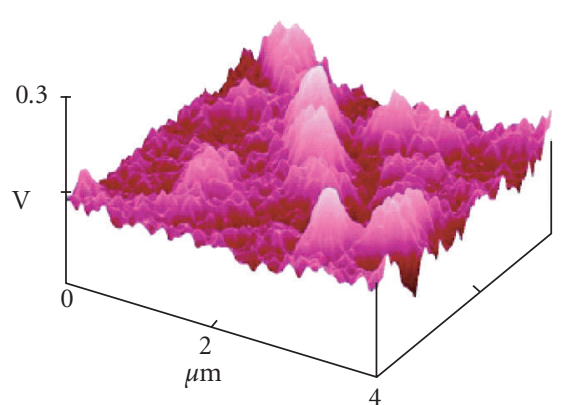

(b)

FIGURE 6: Simultaneous AFM (a) and SNOM (b) of step sites on the (010)-face of sulfanilic acid monohydrate (6) after short exposure to $\mathrm{NO}_{2}$ gas (1:4 mixture with air injected from a $1 \mathrm{~cm}$ distance and left for $1 \mathrm{~min}$ ), showing bright chemical contrast in (b) precisely at the slope sites in the various directions where the product (7) resides.

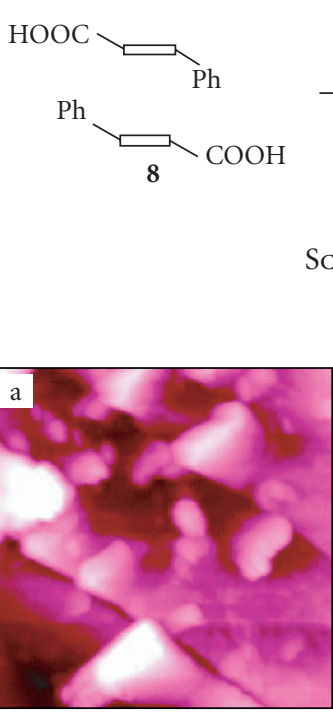

(a)

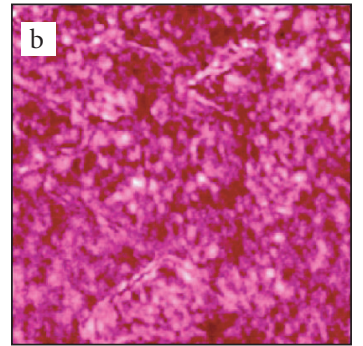

(b)

Figure 7: Simultaneous AFM (a) $(11 \mu \mathrm{m}, \mathrm{Z}$ range $1.5 \mu \mathrm{m})$ and apertureless shear-force SNOM (b) of the (010)-face of $\alpha$-cinnamic acid (8) after $15 \mathrm{~min}$ irradiation at $\lambda>290 \mathrm{~nm}$ showing anisotropically grown features along the cleavage planes direction and only slight local steepness contrast but no chemical contrast.

\section{APPLICATION OF SNOM TO PREHISTORIC OBJECTS}

\subsection{Pyrite from petrification of organic materials, RAMAN SNOM identification.}

Geological fossil stones, such as dolomite, contain pyrite particles from the sulfur content of ancient biological materials upon petrification of the organic material. Figure 11 shows AFM and SNOM with a bright optical contrast on a polished sample. The location of the geode can only be seen in the optical image but not in the AFM scan.

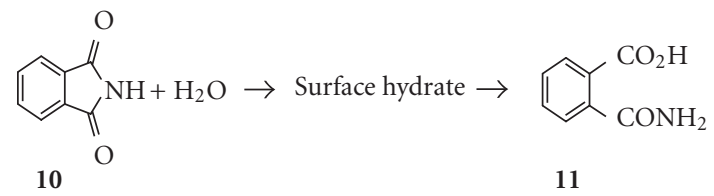

SCHEMe 5

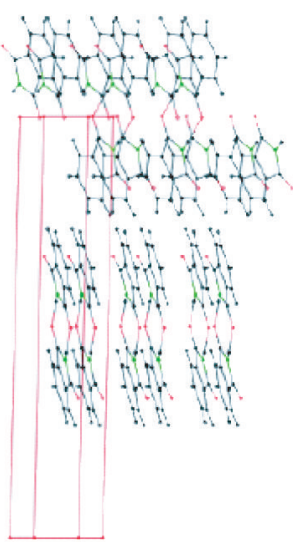

(a)

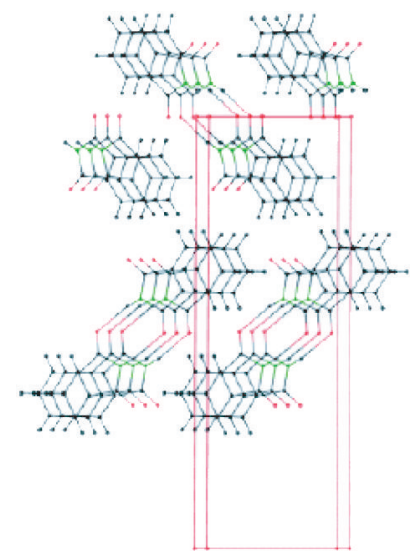

(b)
FIGURE 8: Crystal packing of phthalimide (10) including hydrogen bonds: (a) on (010); (b) on (101); both images are turned around $y$ by $10^{\circ}$ for a better view; the (001)-face is on top in both images.

Clearly, the chemical nature of the material under the bright spot in Figure 11(b) has to be identified by near-field spectroscopy. This is possible by RAMAN SNOM due to the large enhancement factors that are obtained in the shearforce gap of about $5 \mathrm{~nm}$ between very sharp tip and surface, which also enhances the local RAMAN intensity (Figure 12). It is thus possible to collect the local RAMAN spectrum of the highly reflecting geode at or under the polished dolomite sample in Figure 11 with the illuminated ( $488 \mathrm{~nm}$ ) uncoated 


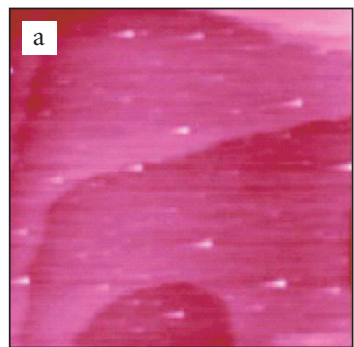

(a) $z=10 \mathrm{~nm}$

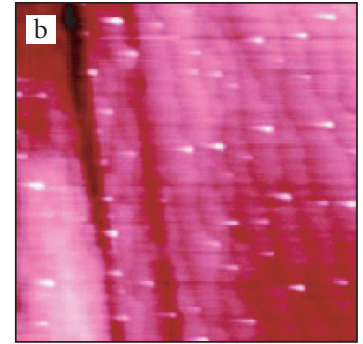

(b) $z=10 \mathrm{~nm}$

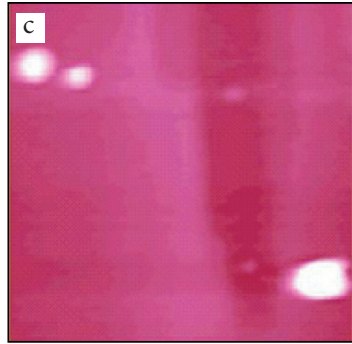

(c) $z=200 \mathrm{~nm}$

FIgURE 9: AFM topographies $(5 \mu \mathrm{m})$ on (001) of phthalimide (10) at room temperature: (a) fresh from acetone; (b) after $2 \mathrm{~d}$ in air at relative humidity of 50-60\%; (c) after $16 \mathrm{~h}$ storage in a vacuum near the saturation pressure of water at room temperature.

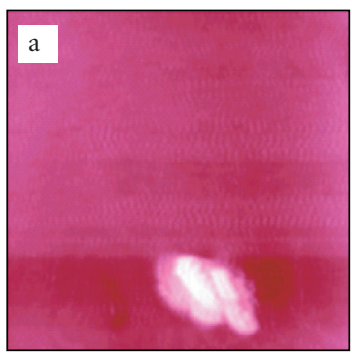

(a)

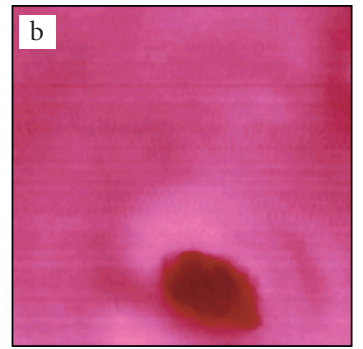

(b)
Figure 10: Shear-force AFM (a) $(5 \mu \mathrm{m}, \mathrm{Z}$ range $400 \mathrm{~nm})$ and apertureless SNOM (b) $(488 \mathrm{~nm})$ of phthalimide (10) after $16 \mathrm{~h}$ in almost saturated water vapor showing negative contrast at the island where the product 11 resides.

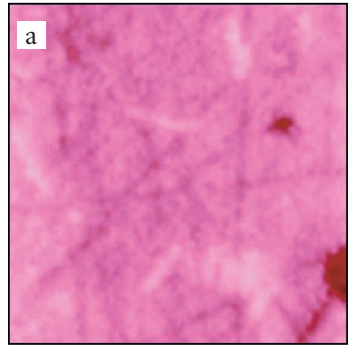

(a)

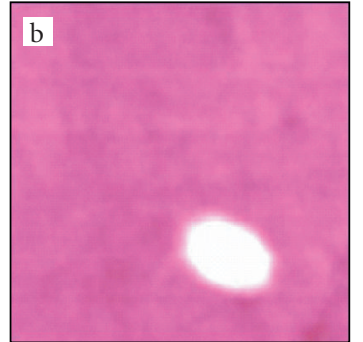

(b)
FIgURE 11: Shear-force AFM (a) ( $5 \mu \mathrm{m}, \mathrm{Z}$ range $100 \mathrm{~nm})$ and chemical contrast by SNOM (b) of a pyrite geode at the surface of polished dolomite, which cannot be traced in the $5 \mu \mathrm{m}$ AFM topography.

sharp tip. The received spectrum after collection of 145 scanlines in the region of the geode is depicted in Figure 12. The spectrum exhibits the known lines of pyrite [16].

Further direct RAMAN SNOM spectra of silicon $\left(519.7 \mathrm{~cm}^{-1}\right)$ and their freshly grown $(5 \mathrm{~nm})$ silica layer $\left(500 \mathrm{~cm}^{-1}\right)$ as well as nonresonant RAMAN SNOM of gal-

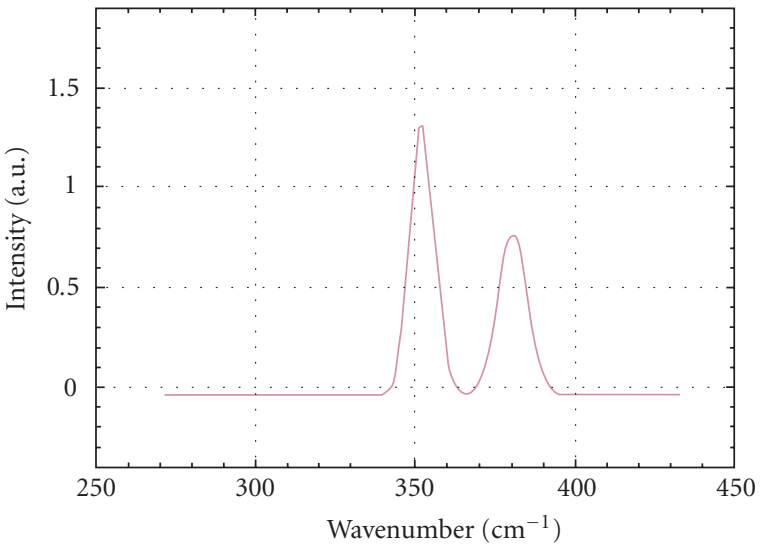

FIGURE 12: Local resonant RAMAN SNOM spectrum of the pyrite geode of Figure 11 (pyrite 145 scanlines) in dolomite; the spectrum is flattened and a baseline subtraction has been executed.

lium nitride $\left[\mathrm{E}_{1}(\mathrm{TO})\right.$ and $\mathrm{E}_{2}$ RAMAN modes at 560.8 and $570.4 \mathrm{~cm}^{-1}$ ] on alumina (subtraction of the RAMAN response of the fiber is essential) with the shear-force apertureless technique (using $488 \mathrm{~nm}$ light) at total collection times of $10 \mathrm{~min}$ have also been reported in [16].

\subsection{Prehistoric bacterium.}

The finely polished dolomite stone (from Dr. Th. Schöberl, Erich-Schmid Institut Leoben, Austria) contains fossil petrified bacteria with diameters of ca. $50 \mu \mathrm{m}$. The AFM (Figure 13(a)) shows a sharp edge due to differences in the abrasive resistance and the SNOM (Figure 13(b)) dark chemical contrast next to the artificial edge contrasts (also the 5 steep protrusions show artificial contrast) in addition to some bright spots that derive from embedded pyrite geodes. The structure in the fossil bacterium part is chemical contrast. Part of it is higher resolved in Figure 14. It has been taken in the area of the petrified bacterium. It clearly contains some still conserved structural details of the bacterium with brighter contrast at the site of the higher more scratch 


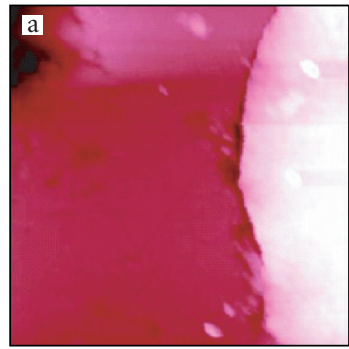

(a)

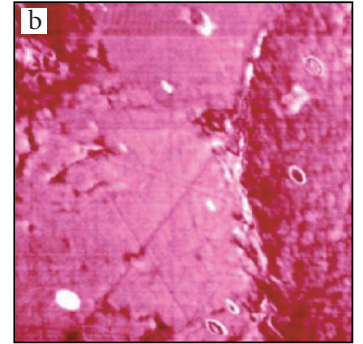

(b)
FIgUre 13: Simultaneous AFM (a) $(20 \mu \mathrm{m}, \mathrm{Z}$ range $2 \mu \mathrm{m})$ and SNOM (b) of a part of a petrified bacterium (dark contrast, right part) in a polished dolomite sample with some pyrite geodes under the surface and dust particles on the surface.

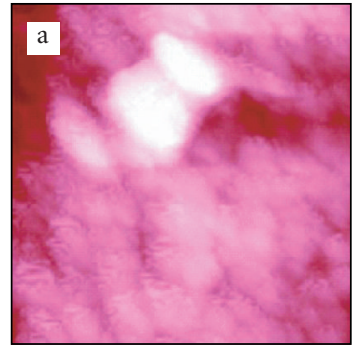

(a)

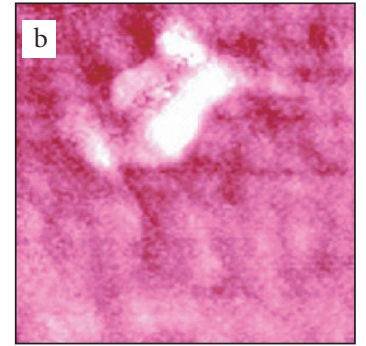

(b)
FIgURE 14: Structure detail of a fossil bacterium in a polished dolomite sample: (a) shear-force AFM ( $5 \mu \mathrm{m}, \mathrm{Z}$ range $300 \mathrm{~nm}$ ); (b) simultaneous SNOM image indicating the chemical difference at the bright contrast site.

resistant feature due to some chemical difference. Further analysis will require local RAMAN SNOM.

\section{APPLICATIONS OF SNOM IN BIOLOGY}

Biological and medicine-related surfaces including microtome cuts are generally rough. They should be studied without further treatment by AFM and SNOM if the highest resolution is desired in order to gain nondestructive subcellular information at the level of organelle structures. Apertureless shear-force SNOM with enhanced reflection back to the sharp fiber is the only technique that can achieve this goal. Confocal microscopy does not detect chemical contrast and it does not reach the possibilities of SNOM on rough surfaces even in the $4-\pi$ configuration. It is, however, useful for preevaluations and positioning. The point-spread function engineering could reach comparably high resolution for flat surfaces, but the versatility of the STED-fluorescence microscope is restricted. Staining changes the biological preparations but it may be helpful for identification purposes of the near-field features in separate SNOM experiments.

\subsection{SNOM on polymer beads.}

Prior to the discussion of soft biological samples, the validity of apertureless shear-force SNOM shall be demonstrated with $430 \mathrm{~nm}$ polymer beads. These are test samples for the applicability of shear-force aperture SNOM in this area. The aggregation of polymer beads has important parallels in the aggregation of biological objects. The chosen beads are copolymers of styrene and acrylamide with uneven distribution of the polar component, which gives rise to polar sites that are of importance in the aggregation from water suspension on mica. Clearly, submonolayer beads have difficult topography due to overhangs below the vertical. Such edge artifact gives rise to highly resolved $(<20 \mathrm{~nm})$ stripes and this could be seen in Figures 15(b), (c). Figure 15(a) images small aggregates and it is clearly seen in the simultaneous SNOM image (Figure 15(b)) that a bright contrast occurs at the site of contact of four or five beads that are not elongated when isolated or embedded in a closed monolayer (Figure 16). Clearly, the more polar and thus more hydrated ends with considerably brighter optical contrast search each other upon aggregation and form the polar center with brighter reflection in these small aggregates.

The arrangement of the beads in closed monolayers is, of course, different. Figure 16(b) indicates that the optical contrast of some beads is considerably brighter than the contrast of others due to different orientation of their polar ends where these accumulate water layers. The interpretations and further use of these results have been detailed in $[17,18]$.

\subsection{SNOM on cryomicrotome cuts of rabbit heart.}

The state-of-the-art cryocut of a rabbit heart, unstained but stabilized with glutaraldehyde, was provided by R. P. Franke, Universität Ulm [19]. Its shear-force AFM and apertureless SNOM easily resolve the organelles despite the remarkable roughness even in a $2 \mu \mathrm{m}$ scan. The image is interpretable at this scan range. Shorter ranges are possible in view of the high resolving capacity but such measurements would require knowledge about the organelle that is searched and hit. Clearly, the AFM surface plot (Figure 17) shows convex features. One of them is identified as lysosome (in front) due to its size and dark optical contrast in the SNOM image. The top views (Figures 17(b), (c)) distinguish the location of various organelles topographically (Figure 17(b)) and by their chemical contrast (Figure 17(c)). At least three types of contrast (dark, medium, and bright) are immediately distinguished. The bright contrast belongs to mitochondria. The differences are obtained without staining. Staining might secure the interpretation in additional SNOM measurements. Furthermore, higher-resolved SNOM traces fine structure of the organelles, the interpretation of which will be subject to histological interpretation that opens a new field for the recognition of biostructures and bioprocesses, but is not outlined here due to the enormous complexity. The histologist is offered the unadulterated organelle image for detailed study with a nondestructive technique. Such information cannot be obtained by aperture SNOM or confocal microscopy. A 


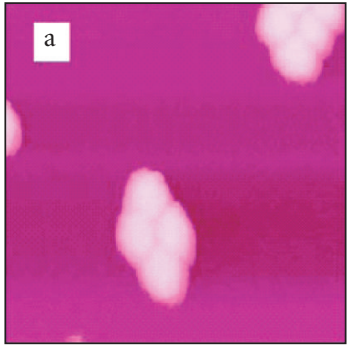

(a)

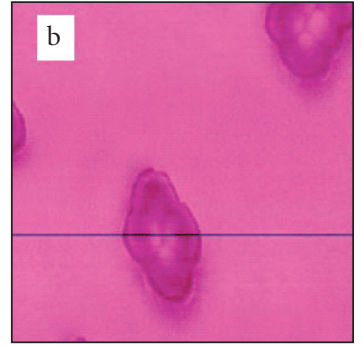

(b)

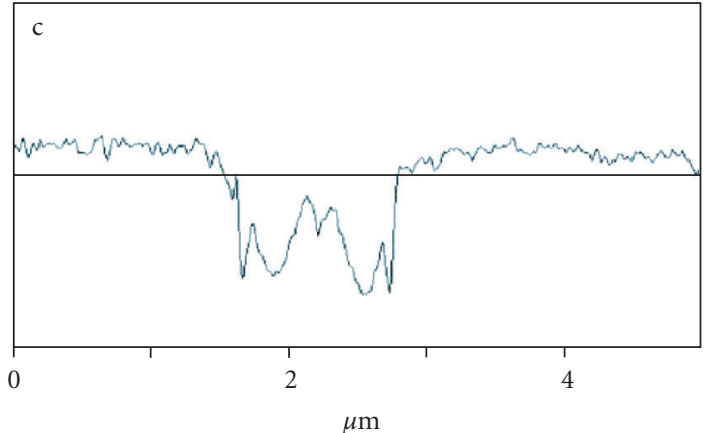

(c)

FIGURE 15: Shear-force AFM (a) and apertureless SNOM (b) of small aggregates of $430 \mathrm{~nm}$ styrene-acrylamide copolymer latices on mica and cross section (c) through the optical contrast showing both highly resolved-stripes contrast at the edges and materials contrast.

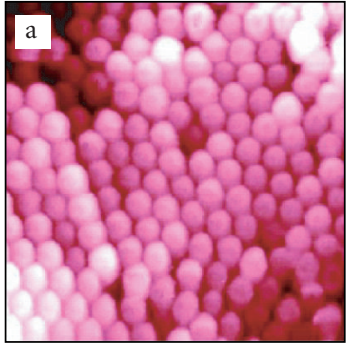

(a)

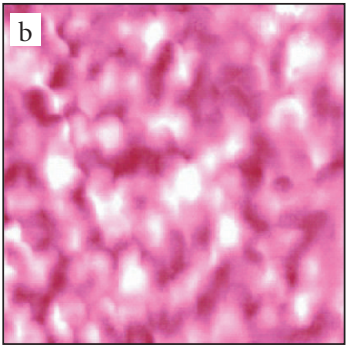

(b)
FIGURE 16: Shear-force AFM (a) and apertureless SNOM (b) of a closed monolayer of $430 \mathrm{~nm}$ styrene-acrylamide copolymer latices on mica showing different brightness of the optical contrast due to different orientation.

major advantage of apertureless SNOM in shear-force distance is the possibility for investigation without extensive preparation. This includes measurements at $0^{\circ} \mathrm{C}$ of fresh cuts and scan areas below organelle size.

A very different region in heart cells is shown in Figure 18. It exhibits the mitochondria with their characteristic lamella structure (Figures 18(a), (b)) and bright reflection (Figure 18(c)). The higher central vesicle with dark optical contrast marks a lysosome. The upper-high lamellae with dark optical contrast are parts of the Golgi apparatus with two distal secretionary Golgi vesicles to the right that give the brightest optical contrast (Figure 18(c)). Clearly, the SNOM image is interpretable, as there is no topographical artifact.

Furthermore, a lysosome (right side with bright optical contrast of the membrane) is clearly seen in Figure 19. The rich-dark optical contrast below the center (Figure 19(b)) and the structured-bright optical contrast left to it indicate large chemical variations in the respective organelles. The features could easily be more highly resolved. The remarkable variations of the optical contrast will certainly find more detailed histological interpretation. This might require combination with common staining techniques.

\subsection{SNOM on stained and unstained shrimp eye preparations.}

The shrimp eye preparations (cryomicrotome cuts stabilized with glutaraldehyde) were provided by P. Jaros, Universität Oldenburg [19]. The organelle scheme of nuclei, reticula cell, rhabdoms, microvilli, tapetum cell, and basal lamina is known [20]. Again, the chemical contrast serves for the identification of the organelles. Figure 20 shows the comparison of a microphoto of a stained sample with the much higher resolved shear-force AFM measurement at very high topography. The sides are too steep for artifact-free SNOM. There is the risk of adulteration due to extensive staining of this preparation.

Lysosomes and mitochondria give the same optical contrast with SNOM as in the case of the rabbit heart if an unstained preparation of the shrimp eye cryomicrotome cut with glutaraldehyde stabilization is measured at much higher resolution in a flat region as in Figure 21. Numerous granulae (containing vision pigments) with dark optical contrast and a lysosome (large ball with structured optical contrast) are resolved and identified by their shape in the surface view (Figure 21(a)) or in the topview (Figure 21(b)), as well as by their optical contrast in the SNOM image (Figure 21(c)). The bright contrast in the optical image is attributed to the mitochondria, which are rich in highly reflecting materials. A complicated array of these and other organelles is recognized.

The power of apertureless SNOM in shear-force distance is even more clearly evidenced by the higher-resolved $2.5 \mu \mathrm{m}$ scan of the lysosome in Figure 22, which images structural details despite its position at a slope (Figure 21(a)). The membrane is bright and the interior shows structural details. The resolution can be further improved in view of the high lateral resolution of this SNOM technique. Interestingly, SNOM shows much more details than AFM. Further 


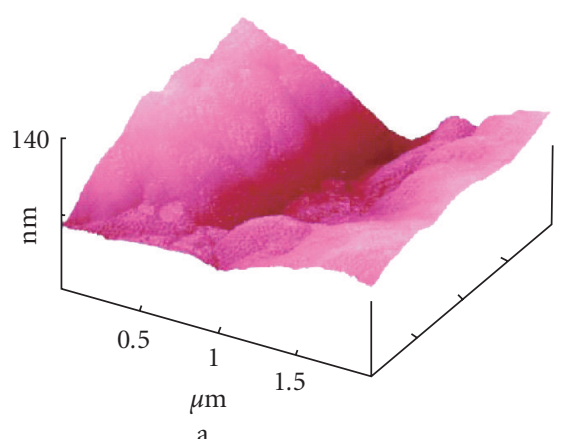

(a)

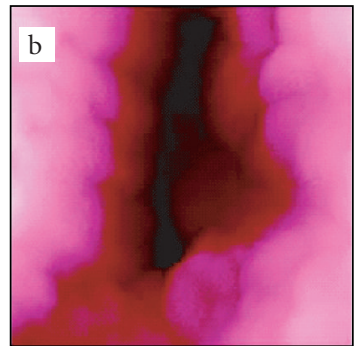

(b)

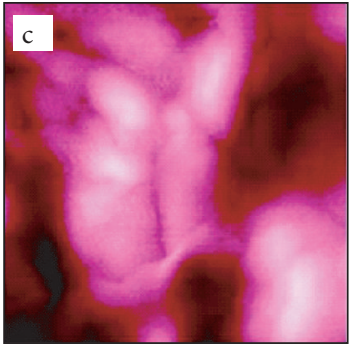

(c)

Figure 17: Surface view (a) and top view shear-force AFM (b) of a cryomicrotome cut from a rabbit heart and simultaneous apertureless SNOM (c) showing a lysosome (bottom, dark optical contrast) and mitochondria (bright optical contrast) next to further organelles.

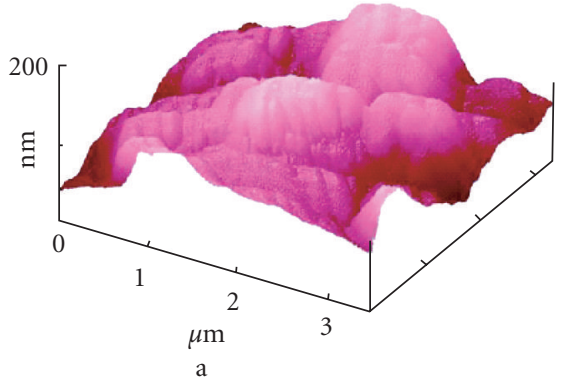

(a)

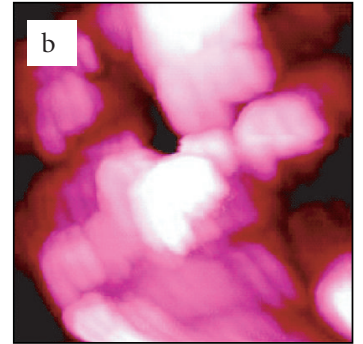

(b)

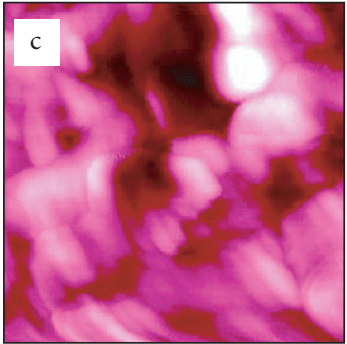

(c)

FIGURE 18: Shear-force AFM (a), (b) and apertureless SNOM (c) of a cryomicrotome cut of a rabbit heart showing lamella structures of mitochondria (bright optical contrast), lamella structures with dark contrast (part of the Golgi apparatus) next to two most brightly reflecting Golgi vesicles (upper part), and a central dark reflection (lysosome).

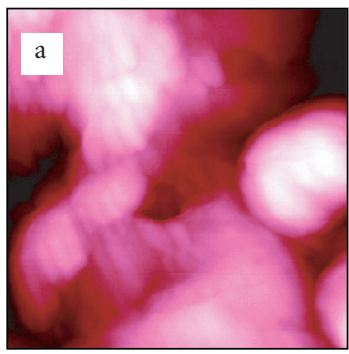

(a)

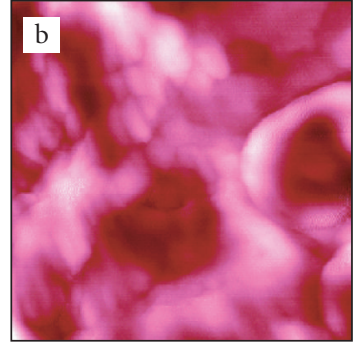

(b)
Figure 19: Shear-force AFM (a) $(3.5 \mu \mathrm{m}, \mathrm{Z}$ range $100 \mathrm{~nm})$ and apertureless SNOM (b) of a cryomicrotome cut of a rabbit heart showing lamella structures and a lysosome (bright membrane and dark interior optical contrast to the right). The various organelles show very diversely structured optical contrast in line with highly varied chemical differences at the corresponding sites.

assignments are the sake of cell biologists. They are offered a robust photophysical tool to study suborganelle structures with unadulterated preparations.

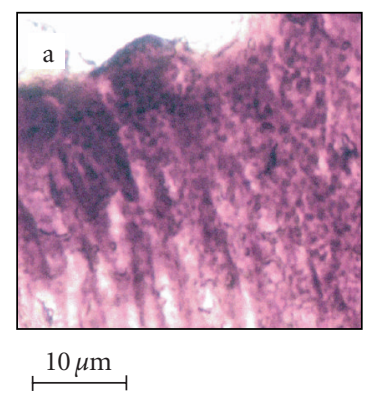

(a)

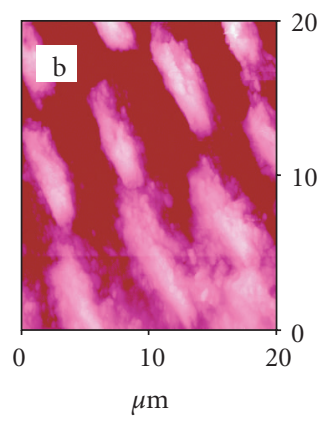

(b)
Figure 20: Microphoto (a) and shear-force AFM (b) of a stained (toluidine blue and osmium tetroxide) shrimp eye in the peripheral region; the $Z$ range in the AFM is $4 \mu \mathrm{m}$.

\section{APPLICATION OF SNOM IN MEDICINE}

Apertureless shear-force SNOM is equally well suited in medicine for the advancement of biocompatibility, implantology (Section 7.1), submicroscopic histology and pathology including oncology, blood storage (Section 7.3), and so on. 


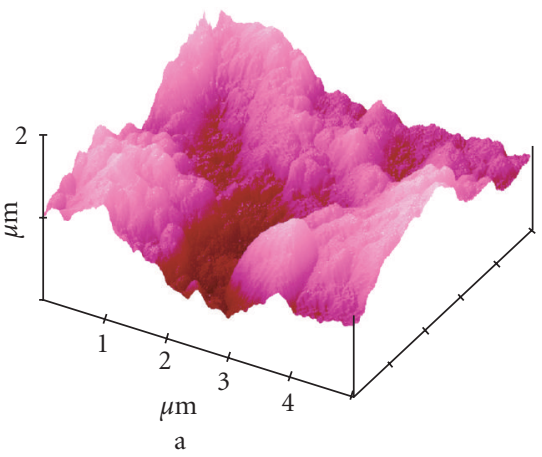

(a)

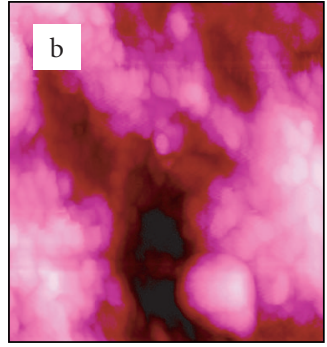

(b)

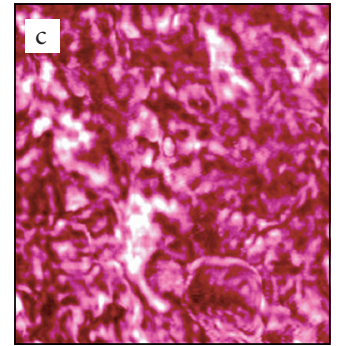

(c)

FIGURE 21: Shear-force AFM (a), (b) and apertureless SNOM (c) of an unstained cryomicrotome cut of a shrimp eye in the peripheral region resolving various organelles by topography and chemical contrast in the optical image.

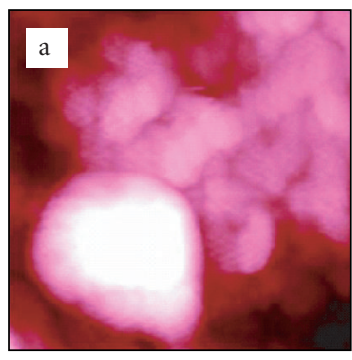

(a)

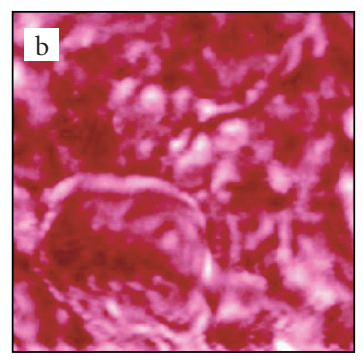

(b)

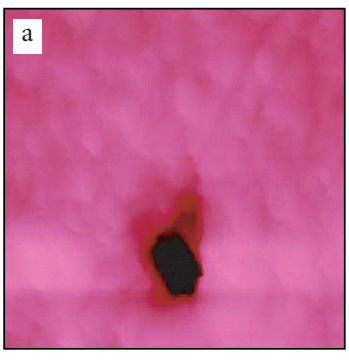

(a)

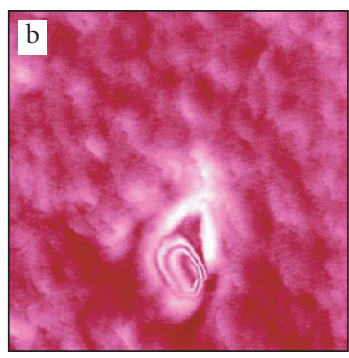

(b)

Figure 22: Higher-resolved (pixel size $5 \mathrm{~nm}$ ) $2.5 \mu \mathrm{m}$ AFM (a) and SNOM image (b) of the lysosome and adjacent organelles in an unstained cryomicrotome cut of a shrimp eye in the peripheral region.

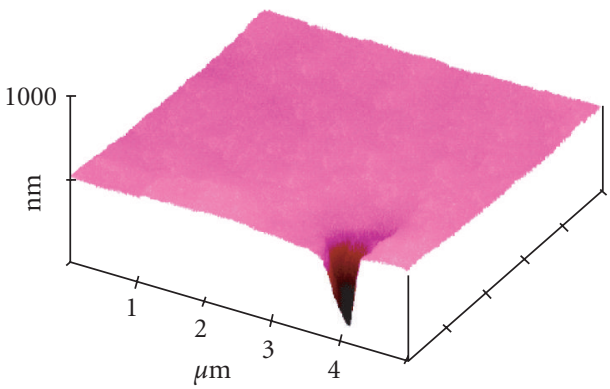

FIgURE 23: Shear-force AFM topography of a human tooth on a microtome cut orthogonal to the tubuli direction showing deep penetration of the sharp tapered tip.

\subsection{SNOM on a human tooth.}

A human tooth was cut with a microtome (sample provided by R. P. Franke, Universität Ulm) [19]. It has been used for a study of the tubuli structure. Shear-force AFM with the sharp-tapered tip is quite successful. The vertical cylinder could be deeply descended without tip breakage (Figure 23).

FIgURE 24: Simultaneous shear-force AFM (a) $(5 \mu \mathrm{m}, \mathrm{Z}$ range $500 \mathrm{~nm}$ ) and SNOM (b): the SNOM image shows both artificial rings at the impossible tube structure site and an interesting chemical contrast at the dark triangle extending further away.

The simultaneous AFM and SNOM images provide unexpected structural results at the tubulus, which are subject to histological interpretation (Figure 24). The rings at the impossible topography (open cylinder) in Figure 24(b) are stripes artifact [6]. The nature of the strongly reflecting material around the triangle and extending into the fissure in Figure 24(b) must be the subject of further histological interpretation. It should be noted that the topographic triangle in Figure 24(a) is probably a rupture result of the microtome cut which might have changed the stressed environment around the edges by putting pressure and redistributing collagen-free dentin from the inner walls of the tubulus. The constituents of intertubulus dentin are hydroxylapatite, mucopolysaccharides, and collagens.

More pronounced microtome ruptures occur at the boundary of enamel and dentin (Figure 25). This type of preparation artifact is more distinctly seen by SNOM than by AFM, notwithstanding the steepness artifact.

Figure 25 shows in both parts very extended ruptures. The enamel (upper part) forms high rims and extends far into the dentin, and there are also deep fissures into the enamel. The rims are due to the different elasticity of the materials. Most prominent in the SNOM image is the edge 


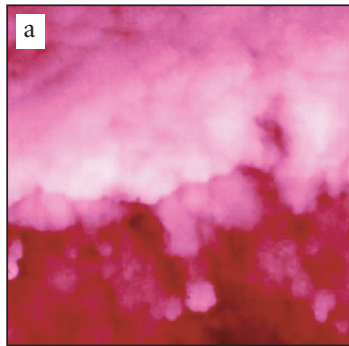

(a)

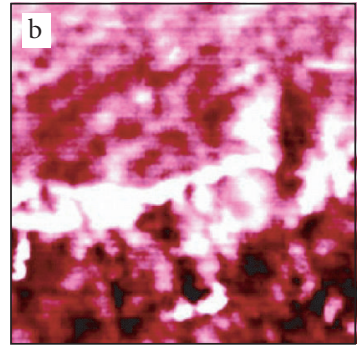

(b)
FIgURE 25: Microtome ruptures at the enamel-dentin boundary as determined by (a) shear-force AFM $(10 \mu \mathrm{m}, \mathrm{Z}$ range $1 \mu \mathrm{m})$ and (b) apertureless SNOM.

artifact at the rim sites. It can however be seen that enamel has a brighter contrast than dentin. The rupture is shown by the dark contrast entering the enamel region. However, some of the interpenetration of the two materials might be natural. Therefore, similar SNOM should be measured at the uncut surface of the tooth in order to clarify this point. Topography that may occur at the natural interface will not be impeding to that endeavor.

\subsection{SNOM in cancer research.}

Subcellular chemical contrast with high and very high local resolution enables screening for distinctive indicators of tumors in the organelles of pathological cells as compared to the corresponding healthy cells. Apertureless shearforce SNOM is well suited while other submicroscopic techniques are unsuitable as there is generally very high roughness in the tissue samples. The high potential for precancerous diagnosis by chemical contrast derives from Rayleigh and RAMAN scattering, fluorescence emission, light absorption, and shear-force efficiency by the site-specific shearforce mechanisms. Cancer prediagnosis is of eminent medicinal importance. Bladder tissue and bladder cancer tissue were cooled with water to $-10^{\circ} \mathrm{C}$, cut, and dried in air. The parallel cuts served for selective staining to mark the cancerous regions. The samples were provided by G. Müller, LMTB, Berlin [19]. Interestingly, the staining of the samples wipes out all pertinent optical information in apertureless shear-force SNOM. This is shown in Figure 26 with scans over 25 and $10 \mu \mathrm{m}$. No chemical contrast is recognizable after HE staining; whereas the appearance of the AFM topography remains largely unchanged by staining, as is evident from the comparison of Figure 26(c) with Figure 27. It is also evident that different sites of biological tissue cuts at the $10 \mu \mathrm{m}$ range have different topography. However, both the unstained and the stained samples exhibit small spheretype features at this range of resolution that seem to be less frequent in the healthy unstained tissue (Figure 27(b)). This latter difference may be not a reliable criterion for cancer diagnosis due to high variability at different sites. More distinct are the differences in the chemical contrast of SNOM.
Figures 28(a), (b), (c) show the topographic variations at three sites for healthy unstained tissue and also the simultaneously recorded SNOM contrast (Figures $28\left(\mathrm{a}^{\prime}\right),\left(\mathrm{b}^{\prime}\right)$, $\left.\left(c^{\prime}\right)\right)$. It is clearly seen in the images of Figure 28 that the topographic variation is indeed very pronounced even in the large range scans. Furthermore, the chemical contrast exhibits dark, medium, and bright spots that are spread over the surfaces and are not very characteristic. The same type of images but of cancerous bladder tissue is shown in Figure 29. The topography of the cancerous tissue at the range of $20 \mu \mathrm{m}$ is not very different in character from the healthy tissue. However, there are distinct differences in the chemical contrast between Figures 28 and 29, which are very useful for the differentiation. It is clearly seen in Figures $29\left(a^{\prime}\right),\left(b^{\prime}\right)$, and $\left(c^{\prime}\right)$ that much more distinct chemical contrast occurs in the form of bright-extended plate-like zones that have frequently sharp edges that seem to fit in with topographic features. Such correspondence is very important and not artificial as the topographic features are in sections of both depressions and hills in the same image. The correspondence identifies cell regions that are highly reflective in the shearforce gap. The high reflectance is assumed to be due to accumulation of metal ions and mitochondria in the rapidly replicating cells. Such plate-like chemical contrast does not occur with healthy tissue (Figure 28). This observation provides a clue for precancer diagnosis when the cells start to increase their properties and these new chances should not be disregarded in oncology. The investigations must be urgently extended to other types of cancer. For example, the breast carcinoma is most frequently diagnosed by the microcalcification at a rather late stage. Our SNOM should be able to easily detect sub-microcalcification in a very early stage, primarily at patients with a genetic risk.

Our proper SNOM for rough surfaces is versatile, robust, and economic. The plate-like chemical contrast is not seen in confocal microscopy. The new diagnostic possibilities profit from the fact that apertureless SNOM in constant shear-force prefers minimal pretreatment of the tissues, which are unstained. Rather, the stained samples had lost the optical contrast altogether (Figure 26).

\section{INDUSTRIAL APPLICATIONS OF SNOM}

Apertureless SNOM in the reflection back to the sharp tip mode for rough surfaces is highly application based because it is reliable and particularly uncomplicated and economic. It should now become state of the art after the basic physical facts [1] and the experimental understanding have been worked out. Application possibilities are manifold and very promising. Actually, first industrial applications have already been successful. For example, existing and new dental alloys have to be checked for their corrosion properties in order to prevent harm to the patients. Electrochemical corrosion models are based on chemical analysis. However, submicroscopic analysis of the surface after corrosion tests must be urgently executed. They are equally important with further implant materials. SNOM provides a new approach by pointing out nanoscopic local differences in the corrosion 


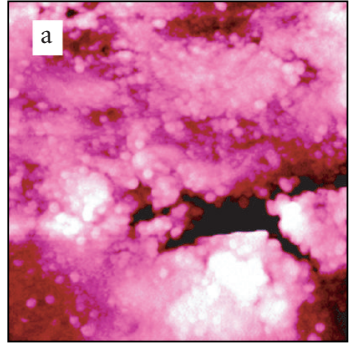

(a)

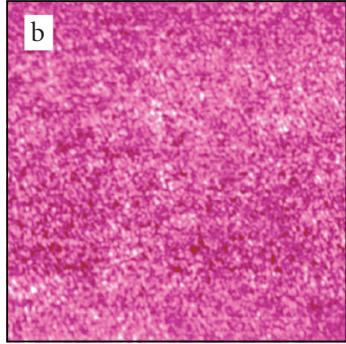

(b)

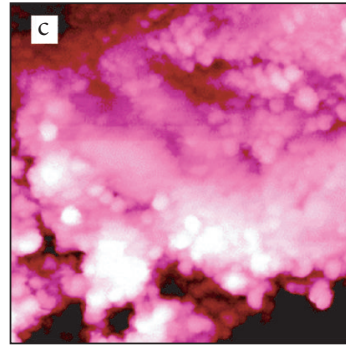

(c)

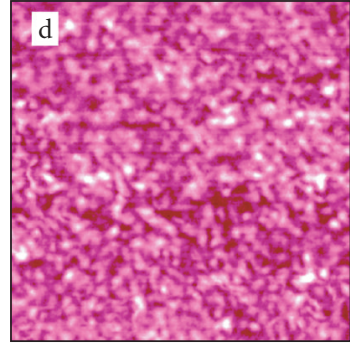

(d)

Figure 26: Shear-force AFM (a), (c) and apertureless SNOM (b), (d) of a HE-stained microtome cut of cancerous bladder cells: (a), (b) $25 \mu \mathrm{m}$ scan; (c), (d) $10 \mu \mathrm{m}$ scan; the $\mathrm{Z}$ range is $2 \mu \mathrm{m}$ in (a) and $1 \mu \mathrm{m}$ in (c).

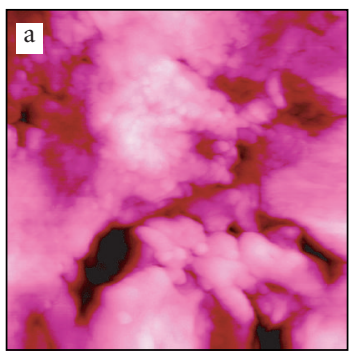

(a)

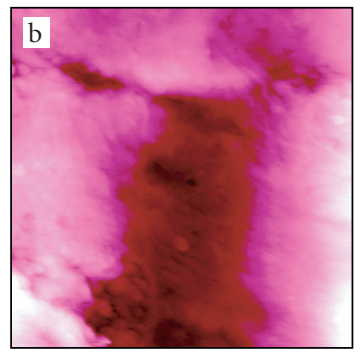

(b)
FIGURE 27: Shear-force AFM ( $10 \mu \mathrm{m}, \mathrm{Z}$ range $1 \mu \mathrm{m})$ of unstained microtome cuts of (a) cancerous bladder tissue and (b) healthy bladder tissue.

process or in biocompatibility by its chemical contrast. Actually, a widely used alloy was eliminated from the market as SNOM indicated the formation of nanoscopic Galvanic elements with pitting corrosion. Furthermore, better alloys that do not exhibit the pitting could be identified as potential substitutes [19]. Further emerging fields of application are nanoparticles, varnish, finish, glazing, dyeing, semiconductors, catalysts, and composites, to name a few. Shear-force apertureless SNOM on rough surfaces complements often used confocal microscopy in various ways due to higher resolution and chemical contrast and by direct comparison with topography. Local SNOM spectroscopy using shear-force gap enhanced fluorescence emission and RAMAN scattering are further promising tools.

\subsection{SNOM on dental alloys.}

The study of SNOM on pristine and corroded dental alloys was part of a governmental project in cooperation with Dr. Liefeith from IBA, Heiligenstadt, Germany. The standardized electrochemical corrosion of different dental alloys provides samples with different SNOM response, ranging from smooth uniform leaching (favorable) to submicroscopic pitting (unacceptable). This is easily detected by shear-force AFM and simultaneous apertureless SNOM. Submicroscopic pitting will occur upon creation of local Galvanic elements. Figure 30 shows the results of a cobalt-containing abraded alloy using AFM and SNOM before and after high electrochemical load. The degree of abrasion in Figure 30(a) is typical for direct use at the dentist site. The sharp edges enforce stripes artifacts in the SNOM but there is no additional chemical contrast in Figure 30(b) and neither so in Figure 30(d) even though the topography in Figure 30(c) has changed upon standard corrosion. This appears to be a good result if chemical analysis of the leached metal ions is also acceptable. Similar favorable results were also obtained with polished or mold samples of the same alloy. Such judgment is not available by confocal microscopy or XPS and other techniques.

Totally different is the behavior of a widely used nickelcontaining alloy that gave the results of Figure 31. The edges in the pristine-abraded alloy (Figure 31(a)) are sharp and give the unavoidable artificial optical contrast in addition to some very small dark points that are chemical contrast (Figure 31(b)). After standard electrochemical treatment, the edges disappear and several craters are formed (Figure 31(c)). Most importantly, the SNOM image shows chemical contrast (Figure 31(d)) indicating different metallic composition at the different sites and that means local Galvanic elements are formed. Clearly, submicroscopic pitting occurs. This unacceptable material was immediately withdrawn from the market and is not further used as a dental alloy after these results.

Another abraded copper-containing dental alloy corroded smoothly and gave the results of Figure 32. Again we have sharp edges (Figure 32(a)) and an artificial stripes contrast in the SNOM image (Figure 32(b)). The additional high feature in Figure 32(a) is responsible for a strong chemical contrast in Figure 32(b) that can be clearly identified next to the artificial stripes.

At low electrochemical load, the additional topography and the corresponding chemical contrast disappear. Only the stripes remain (not shown). Most importantly, after high electrochemical load, the topography changes significantly (Figure 32(c)), but no contrast at all is discernable in the SNOM image (Figure 32(d)). This appears to be a favorable dental alloy as far as the corrosion behavior is concerned. 


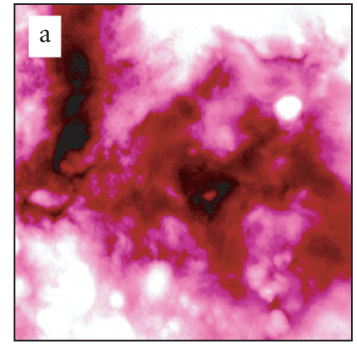

(a)

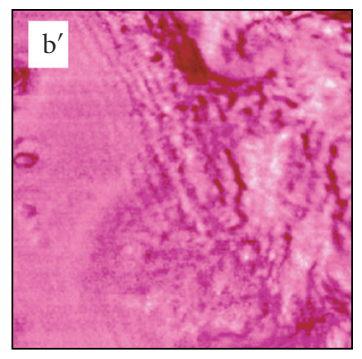

$\left(\mathrm{b}^{\prime}\right)$

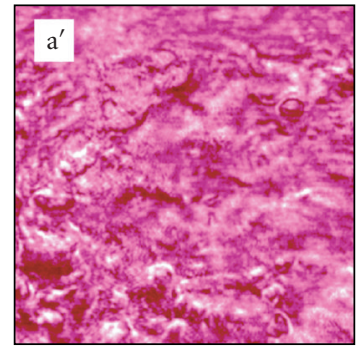

$\left(a^{\prime}\right)$

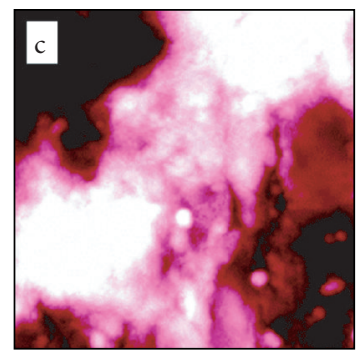

(c)

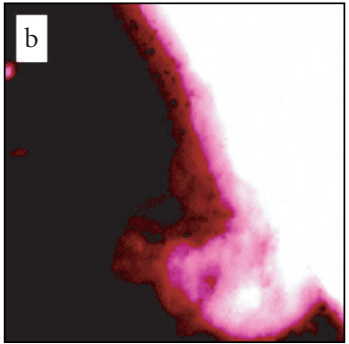

(b)

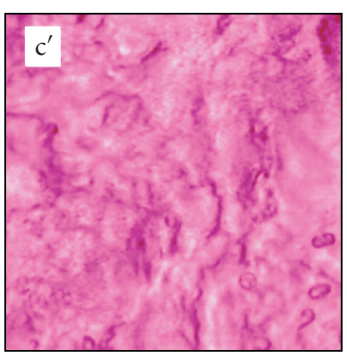

$\left(c^{\prime}\right)$

FIgURE 28: Shear-force AFM topographies $(25 \mu \mathrm{m}, \mathrm{Z}$ range $1 \mu \mathrm{m})$ at three different sites (a), (b), (c) and the corresponding optical contrast $\left(a^{\prime}\right),\left(b^{\prime}\right),\left(c^{\prime}\right)$ of apertureless SNOM on the rough surfaces of healthy bladder tissue.

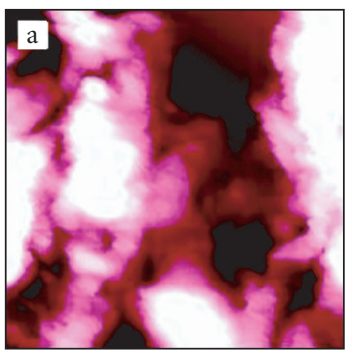

(a)

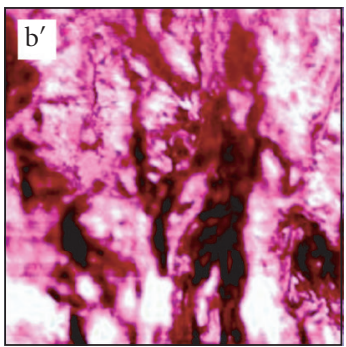

$\left(b^{\prime}\right)$

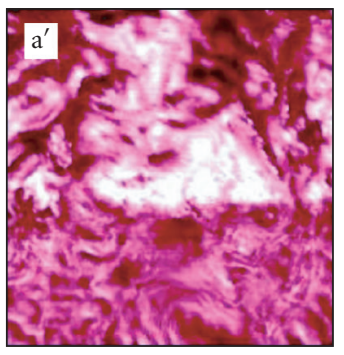

$\left(a^{\prime}\right)$

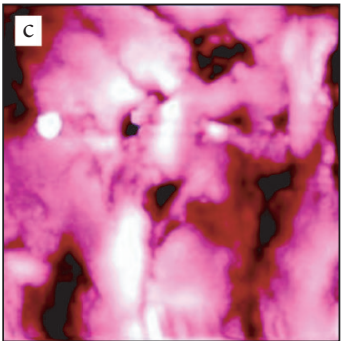

(c)

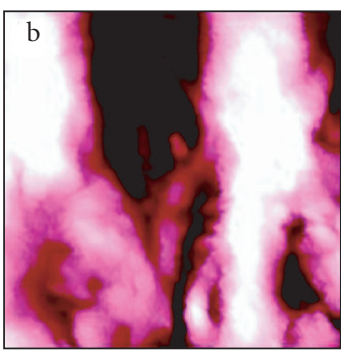

(b)

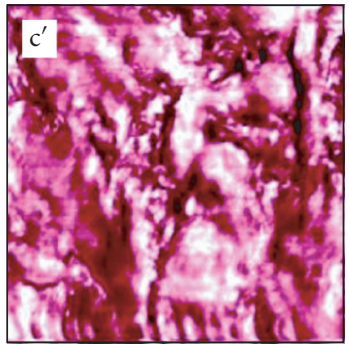

$\left(c^{\prime}\right)$

Figure 29: Shear-force AFM topographies $(20 \mu \mathrm{m}, \mathrm{Z}$ range $1 \mu \mathrm{m})$ at three different sites (a), (b), (c) and the corresponding optical contrast $\left(a^{\prime}\right),\left(b^{\prime}\right),\left(c^{\prime}\right)$ of apertureless SNOM on the rough surfaces of cancerous bladder tissue showing plate-like bright contrast areas.

Numerous further alloys have been similarly studied under various conditions of corrosion [19] and classified. Hence, a reliable photophysical technique is available now that is superior to recent approaches. A major feature of the reliable technique (with high enhancement factors that are typical for metals) is the possibility to distinguish artifacts from impossible topography and genuine materials contrast in the same image.

Our procedure has already found due interest in the study of high-grade steel [21] (100 nm resolution claimed, 


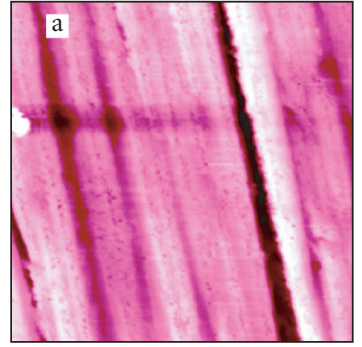

(a)

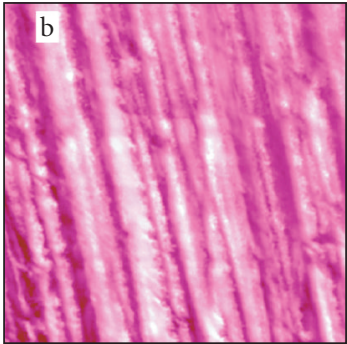

(b)

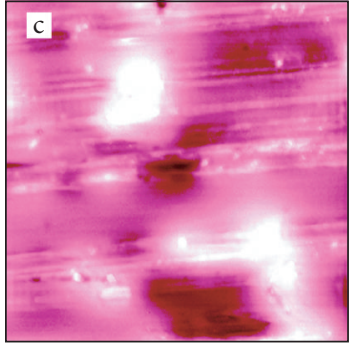

(c)

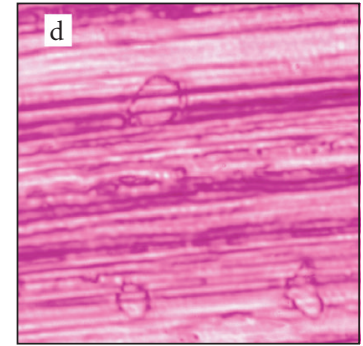

(d)

Figure 30: Simultaneous shear-force AFM (a) $(10 \mu \mathrm{m}, \mathrm{Z}$ range $1 \mu \mathrm{m}),(\mathrm{c})$ (Z range $600 \mathrm{~nm}$ ), and apertureless SNOM (b), (d) of an abraded cobalt alloy: (a) and (b) before, (c) and (d) after standard electrochemical load.

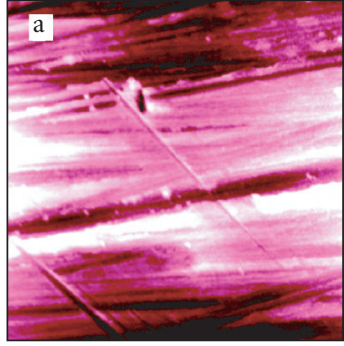

(a)

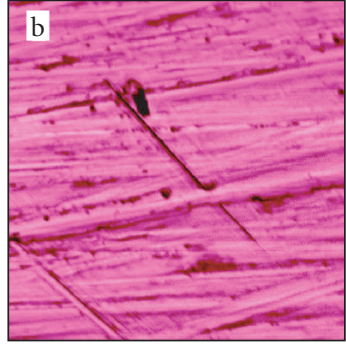

(b)

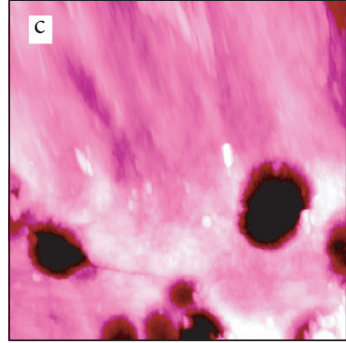

(c)

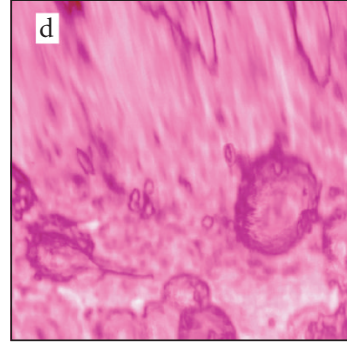

(d)

FIGURE 31: Simultaneous shear-force AFM (a) (10 $\mu \mathrm{m}, \mathrm{Z}$ range $300 \mathrm{~nm})$, (c) (Z range $1.6 \mu \mathrm{m})$, and apertureless SNOM (b), (d) of an abraded nickel alloy: (a) and (b) before, (c) and (d) after electrochemical load, the optical images indicating nanoscopic pitting.

but unfortunately not materials contrast, and the optical images show typical signs of the classified artifacts).

\subsection{SNOM on glazed paper.}

Paper glazing is an important industrial task. Various finely divided minerals are applied in the glazing process. The success depends on numerous parameters. It is of high industrial interest to study the results by monitoring the submicroscopic roughness and the distribution of the materials for optimizing and saving costs of materials in the glazing process. Two suitable technical samples of different roughness are chosen for demonstration of the success with SNOM on the very rough surfaces for this application. It can be seen in the surface views and in the topviews of Figures 33 and 34 how different particles with different near-field reflectivity combine to the total surface. The identification of the particles at the various sites can be obtained from local RAMAN SNOM (Section 4.1). Any added fluorescent components can be located by local fluorescence SNOM. Clearly, such knowledge helps the designer in the optimization of the glazing procedure. The sample for Figure 33 was glossier and felt smoother than the one for Figure 34.

\subsection{SNOM on blood bags.}

The performance of blood bags is of enormous economic importance. AFM measurements at flat parts of commercial highly textured fresh blood bags indicate little change of the submicroscopic (100 $\mathrm{nm}$ range) surface after treatment with physiological sodium chloride solution. However, after short- or long-term storage of blood, the surface changes considerably [19]. This fact is of importance for the allowable storage time of native blood, and submicroscopic investigations are badly required for medicinal and economic reasons. While nanoindentation testing for hardness and elasticity modulus of the bag (14 MPa and $60 \mathrm{MPa}$, respectively) and of the deposits (16 $\mathrm{MPa}$ and $31 \mathrm{MPa}$, respectively) exhibits large differences [19], more detailed information is obtained by the chemical contrast of SNOM measurements with uncoated tips at constant shear-force distance. A fresh blood bag at flat or purposely rough surface does not exhibit SNOM contrast (Figure 35). That means that any topography found in a blood bag that gives SNOM contrast must be a deposit out of the blood. The measurements with the charged blood bags are shown here for the flattest regions that could be found. Organic deposits give chemical contrast at blood bags that were filled with blood for less than one week (Figure 36). It can be clearly seen that deposition is widespread with a brighter contrast at the shallow depression. The chemical contrast proves deposition of blood components to the surface of the blood bags that have not previously been detected, because the hardened solid deposits do not exceed $100 \mathrm{~nm}$ widths. It is still unclear if such deposition is helpful or detrimental for the allowable storage time of native blood. However, further studies are essential 


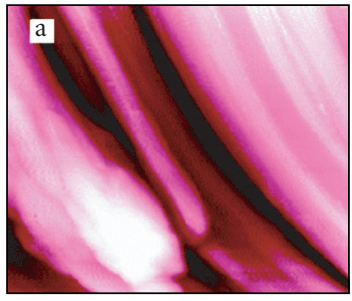

(a)

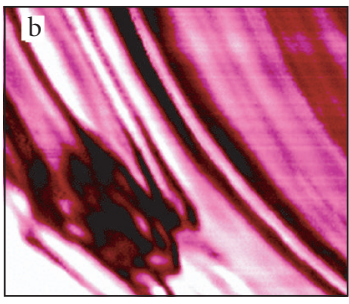

(b)

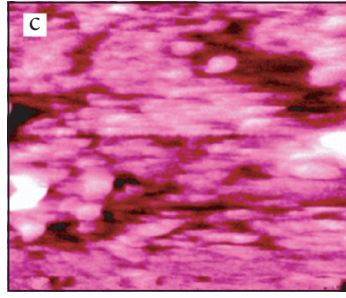

(c)

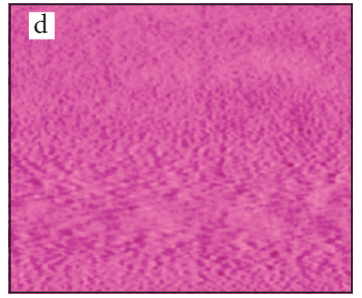

(d)

Figure 32: Simultaneous shear-force AFM (a), (c) (10 $\mu \mathrm{m}, \mathrm{Z}$ range $400 \mathrm{~nm}$ ) and apertureless SNOM (b), (d) of an abraded copper alloy: (a) and (b) before, (c) and (d) after standard electrochemical load, securing very uniform corrosion as judged by lack of SNOM contrast.

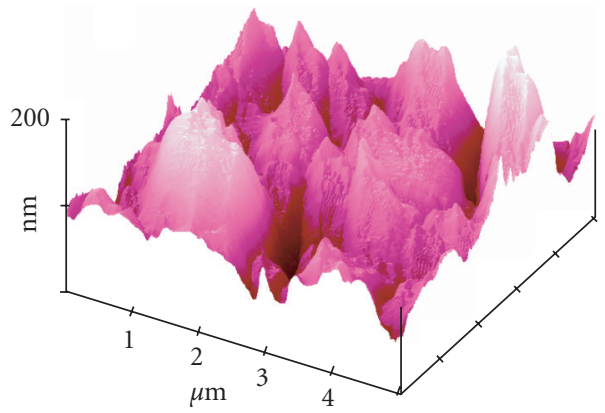

(a)

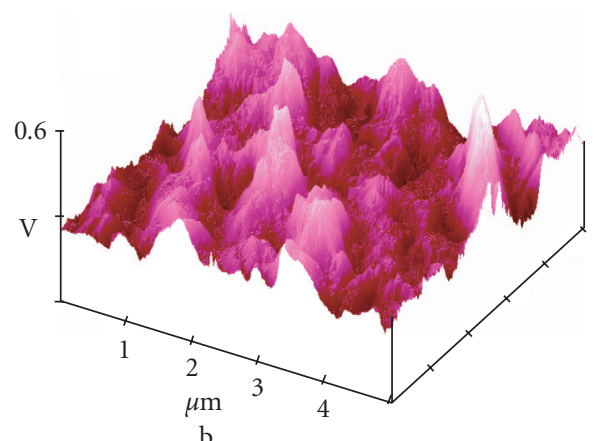

(b)

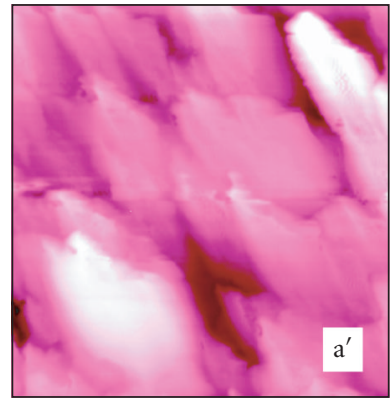

$\left(a^{\prime}\right)$

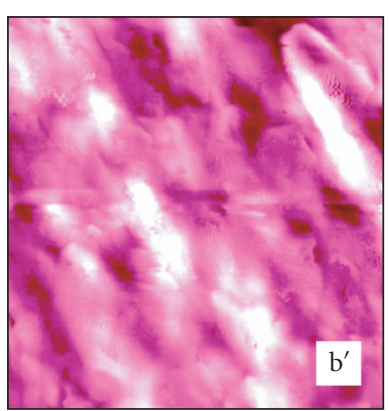

$\left(b^{\prime}\right)$

FIGURE 33: Shear-force AFM (a), ( $\left.a^{\prime}\right)$ and apertureless SNOM (b), ( $\left.b^{\prime}\right)$ of a glossy glazed paper showing roughness and distribution of high, medium, and low optical contrast.

because all chances for safe extension of blood storage must be followed.

More distinct and localized is the chemical contrast of expired blood bags (Figure 37). Again it appears that the brightest contrast is obtained at depression sites, but not uniformly.

\subsection{Quality assessment of metal sol particles for SERS by SNOM.}

Suppliers of silver and gold sols for surface-enhanced RAMAN spectroscopy (SERS) must secure that their prepa- rations will be pure and suitable for the desired large enhancements. Apertureless shear-force SNOM with uncoated tips is the proper analysis technique. Figure 38 shows particles of different sizes in a commercial silver sol preparation after drying on a microscope slide without further treatment. The topography cannot decide if the larger particles are aggregates or impurities. However, SNOM does immediately prove that they are impurity. It further shows that the small particles are not uniform: some exhibiting bright, others medium contrast. Figure 38 is also a good example for proving the SNOM image being free of topographic artifacts: different contrast at positive topographies with 


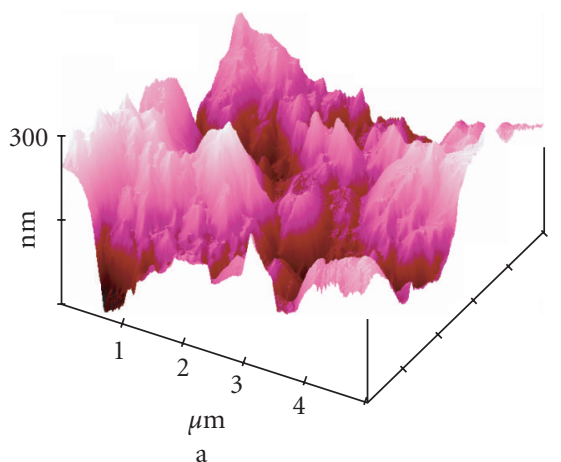

(a)

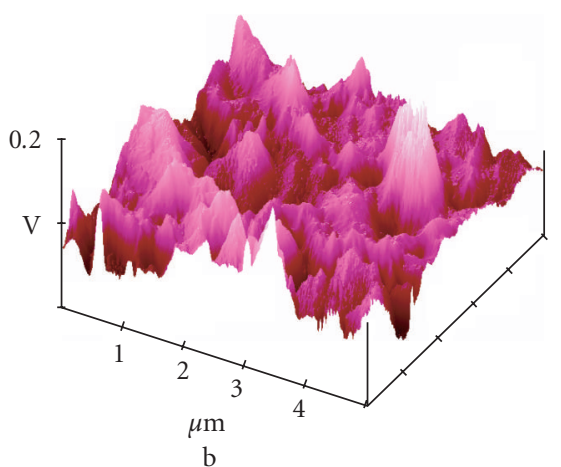

(b)

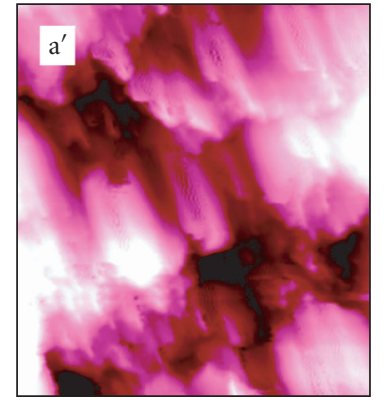

$\left(a^{\prime}\right)$

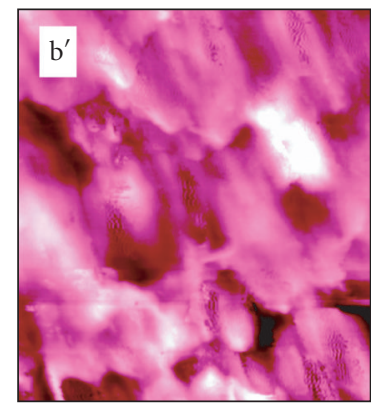

$\left(b^{\prime}\right)$

FIGURE 34: Shear-force AFM (a), ( $\left.a^{\prime}\right)$ and apertureless SNOM (b), ( $\left.b^{\prime}\right)$ of a less glossy glazed paper showing roughness and distribution of high, medium, and low optical contrast.

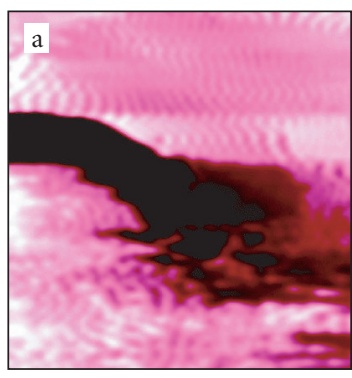

(a)

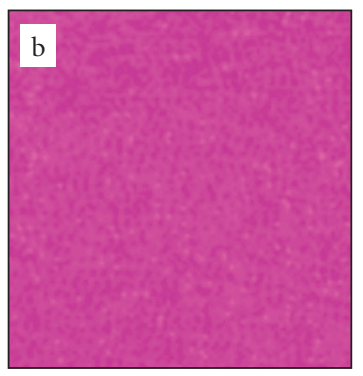

(b)
FIgUre 35: Shear-force AFM (a) $(670 \mathrm{~nm}, \mathrm{Z}$ range $500 \mathrm{~nm})$ and simultaneous apertureless SNOM (b) of a pristine blood bag surface purposely at an unusually rough site showing no optical contrast despite the topography due to homogeneous composition.

different chemical contrast is featured. The chemical nature of the different particles must be clarified by local RAMAN SNOM (Section 4.1).

The attainable enhancement of SERS experiments relies severely on the quality of the colloidal particles. An actually inactive commercial gold sol with nominal $50 \mathrm{~nm}$ particles is

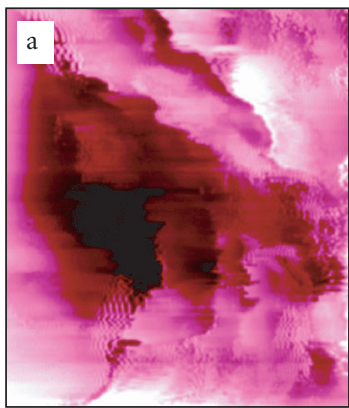

(a)

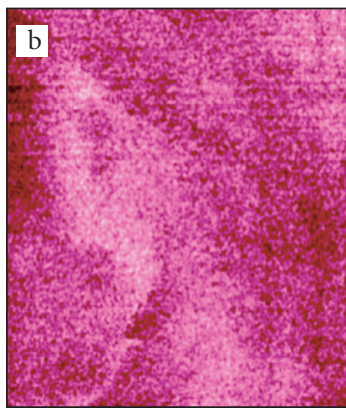

(b)
Figure 36: Shear-force AFM (a) $(800 \mathrm{~nm}, \mathrm{Z}$ range $200 \mathrm{~nm}$ ) and apertureless SNOM (b) of a shortly charged blood bag surface at a particularly flat site showing widespread optical contrast.

analyzed in Figure 39. AFM and SNOM disclose large grains (Figure 39(a)) with dark chemical contrast (Figure 39(b)), which is impurity. Furthermore the higher-resolved images (Figures 39(c), (d)) in an impurity-free region exhibit 400-500 nm wide particles of different heights that are too large. Furthermore, only the higher and regular spheres give 


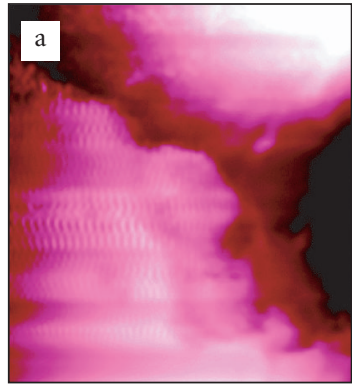

(a)

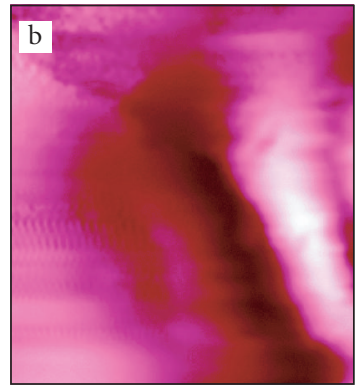

(b)

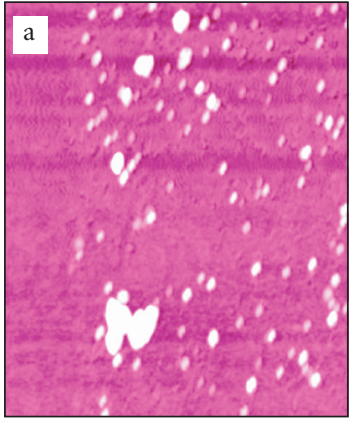

(a)

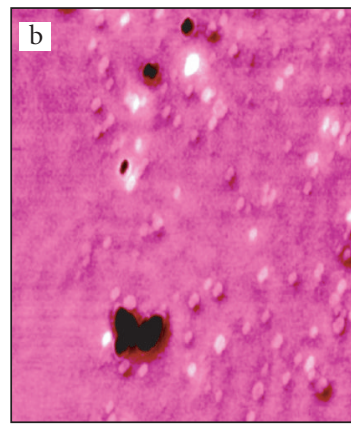

(b)
Figure 37: Shear-force AFM (a) $(500 \mathrm{~nm}, \mathrm{Z}$ range $100 \mathrm{~nm}$ ) and apertureless SNOM (b) of an expired blood bag surface at a particularly flat site showing widespread and pronounced localized optical contrast.

the bright chemical contrast that is expected for gold. None of the specifications is met in this preparation.

Checking every charge of the rather delicate gold sols with shear-force apertureless SNOM prior to sale and purchase is easy, economic, and necessary. The SERS applicant will want to control the stability of the sol prior to the experiment by using this reliable technique.

\subsection{Diffusion coefficients in heat-transfer textile fiber dyeing and light fastness.}

Important technical tasks in textile dyeing include the determination of diffusion coefficients and on-site light fastness. For "real world" samples that have strong irregular topographies, the only technique of choice is the apertureless SNOM for rough surfaces in the back to the fiber reflection in constant shear-force gap configuration. Also the collection of the emitted light from fluorescent dyes by the illuminating sharply tapered tip in shear-force distance is enhanced and best suited for technical or biological applications that must avoid topographic artifacts [16]. Furthermore, this technique is by far less complicated and by far more economic than any other SNOM technique. The industrially applied fluorescing disperse dyes 12-14 of the merocyanine type (Scheme 6) were used in a high-temperature exhaust or heat-transfer dyeing procedure for even or ring coloration of polyester textile fibers. These were embedded into an appropriate resin (Technovit 7100, Heraeus Kuzer Ltd, or Agar 100, Lannet Ltd.), cut with a microtome, and subjected to shear-force apertureless SNOM while eliminating the primary light by an edge filter OG 515 (transmitting from $506 \mathrm{~nm}$ onward; Schott, Germany) in front of the detector for removal of all primary $488 \mathrm{~nm}$ light.

Figure 40 shows the AFM and fluorescence SNOM of the microtome cut with dye $\mathbf{1 2}$. It is clearly seen that the polyester fiber is several $\mu \mathrm{m}$ down due to different hardness and elasticity of the materials. Nevertheless, the fluorescence is evenly strong over the whole area but only at the area of the polyester fiber and not at the slopes, which consist of uncol- ored resin. Furthermore, the minor deficiencies of the very deep AFM are not reproduced in the optical contrast (perspective images in [16]).

SNOM of another polyester fiber evenly colored with dye 13 and embedded in soft Technovit 7100 shows again homogeneously distributed fluorescence (Figure 41(b)) over the fiber cross-section in a skew microtome cut (Figure 41(a)). The slight reduction of light intensity in the deep hole $(3 \mu \mathrm{m}$ wide, $800 \mathrm{~nm}$ deep) and the upper-right corner appear to relate with the unsuitable topography.

The light fastness of dye $\mathbf{1 3}$ has been studied by its fluorescence bleaching in the SNOM. Figure 42 shows the effect of 10 min scanning in a $5 \times 5 \mu \mathrm{m}^{2}$ area with the uncoated illuminated tip $(1.6 \mathrm{~mW})$ giving a $6 \times 6 \mu \mathrm{m}^{2}$ area of photobleaching in the optical image that cannot be seen in the topography. This is a clear technical application for the determination of relative light fastness of dyes in the actual fabrics of interest by large scale SNOM that works well despite very high topography within the scan. This application of SNOM will help designers to improve their products based on clearcut data.

By using the resin Agar 100, the ring dyed-polyester fiber with dye $\mathbf{1 3}$ comes up in the microtome cut as shown in Figure 43(a). The ring dyeing can be seen in both orientations of the textile fiber (Figure 43(b)). The very high topography of an impurity in the center does not give an artifact in the optical image. The lying fiber is flatly cut. The analysis of the near-field fluorescence intensity curve (Figure 43(c)) offers a very easy and versatile new possibility for the determination of the technically important diffusion coefficient of the particular dye in the heat-transfer dyeing process at the actual object rather than in tedious determinations at model systems. The profiles of the near-field fluorescence intensity in Figure 43(c) are fitted to Fick's second law of diffusion, which is numerically solved by using the tabulated values of the "error function" in the usual way. The resulting D-values (diffusion coefficient) are found constant over the complete curve starting at the maximum (right curve chosen). This indicates that the near-field fluorescence intensity is 


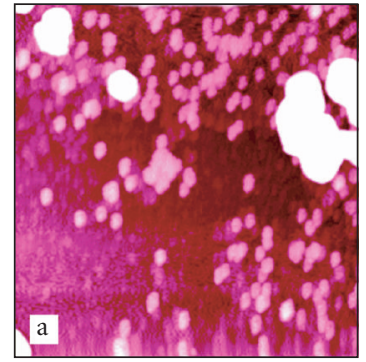

(a)

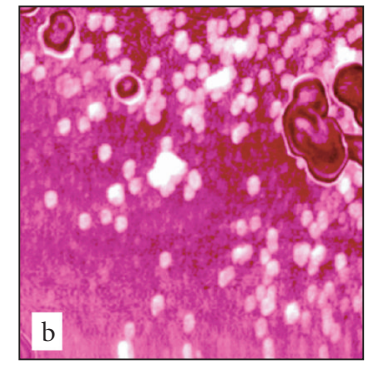

(b)

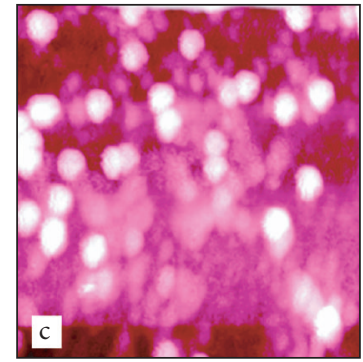

(c)

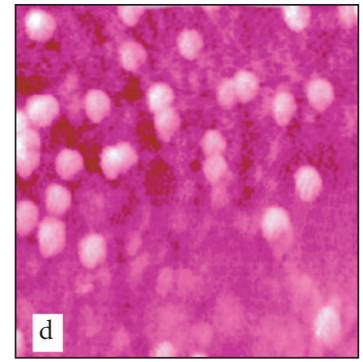

(d)

FIGURE 39: Shear-force AFM (a) $(10 \mu \mathrm{m}, \mathrm{Z}$ range $300 \mathrm{~nm})$, (c) $(5 \mu \mathrm{m}, \mathrm{Z}$ range $200 \mathrm{~nm})$, and apertureless SNOM (b), (d) of a dried SERSinactive au sol on a microscope slide.

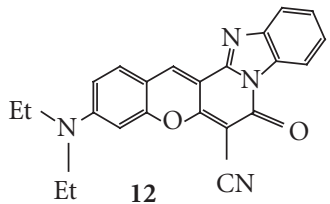<smiles>Nc1c(Oc2ccccc2)cc(O)c2c1C(=O)c1ccccc1C2=O</smiles><smiles>CCN(CC)c1ccc2cc3c(oc2c1)c(C#N)c(=N)n1c2ccccc2nc31</smiles>

SCHeMe 6

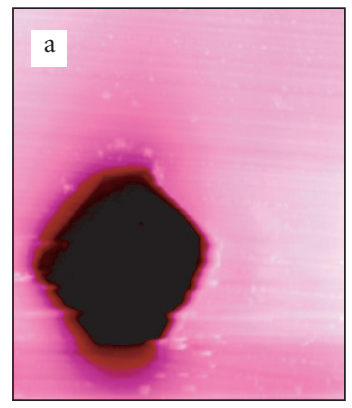

(a)

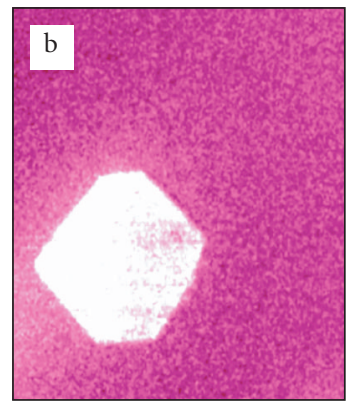

(b)

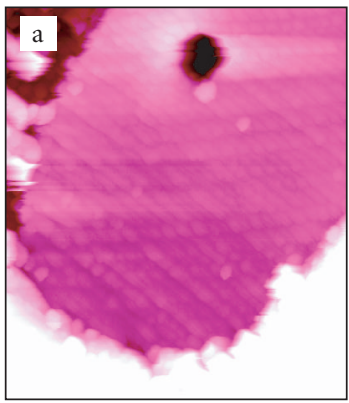

(a)

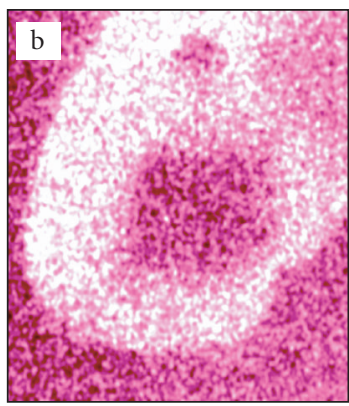

(b)
Figure 40: Simultaneous shear-force AFM (a) $(50 \mu \mathrm{m}, \mathrm{Z}$ range $3 \mu \mathrm{m}$ ) and fluorescence SNOM (b) of a microtome cut surface of an evenly dyed polyester fiber (dye 12) in Technovit 7100 resin.

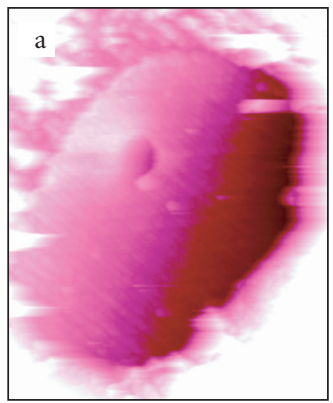

(a)

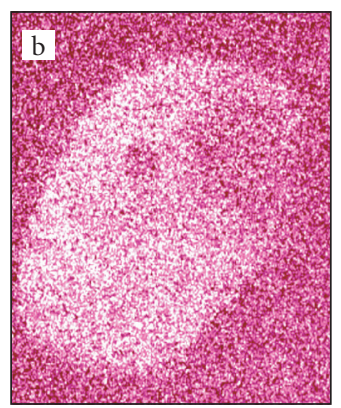

(b)
FIGURE 41: Simultaneous shear-force AFM (a) $(25 \mu \mathrm{m}, \mathrm{Z}$ range $5 \mu \mathrm{m}$ ) and fluorescence SNOM (b) of a microtome cut surface of an evenly dyed polyester fiber (dye 13) in Technovit 7100.
Figure 42: Simultaneous shear-force AFM (a) $(20 \mu \mathrm{m}, \mathrm{Z}$ range $5 \mu \mathrm{m}$ ) and fluorescence SNOM (b) of a microtome cut surface of an evenly dyed polyester fiber (dye 13) in Technovit 7100 after $10 \mathrm{~min}$ illuminated scanning in a $5 \times 5 \mu \mathrm{m}^{2}$ area of the dyed fiber showing local fluorescence bleaching.

proportional to the local dye concentration. The absolute value of the diffusion coefficient at the known dyeing temperature is calculated by using the dyeing time of $30 \mathrm{~min}$ and results in $\mathrm{D}_{115^{\circ} \mathrm{C}}=4.7 \cdot 10^{-11} \mathrm{~cm}^{2} \mathrm{~s}^{-1}$ for the dye 13 . This value is consistent with similar values in the literature [16].

A further ring dyeing with dye $\mathbf{1 4}$ gives closely related results. Figure 44 has the polyester fiber up in Agar 100 resin and clearly indicates the ring coloration. The images (Figures $44(\mathrm{c})$ and (d)) are added in order to show that nonfluorescing topographic impurities do indeed not affect the SNOM contrast in shear-force apertureless SNOM. 


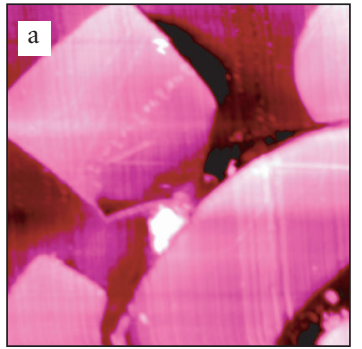

(a)

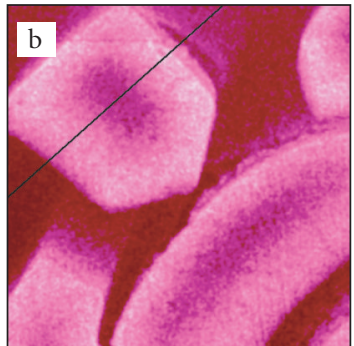

(b)

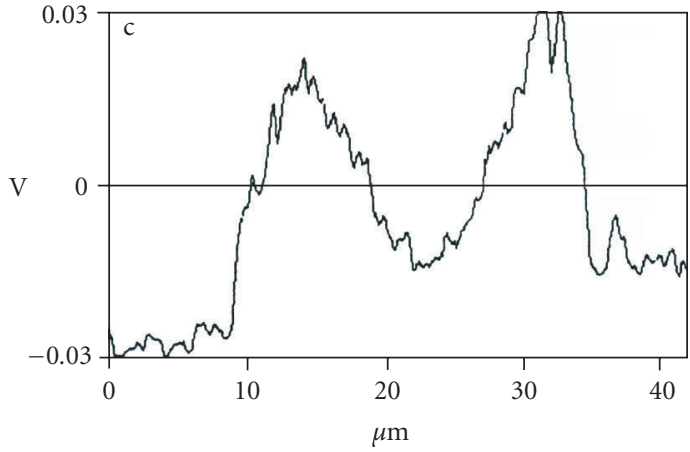

(c)

FIGURE 43: Simultaneous shear-force AFM (a) $(50 \mu \mathrm{m}$, Z range $1 \mu \mathrm{m})$ and fluorescence SNOM (b) of a microtome cut surface of a polyester fiber (dye 13) embedded in Agar 100, showing ring dying in the optical contrast and the cross section (c) through the fluorescence intensity as indicated in (b).

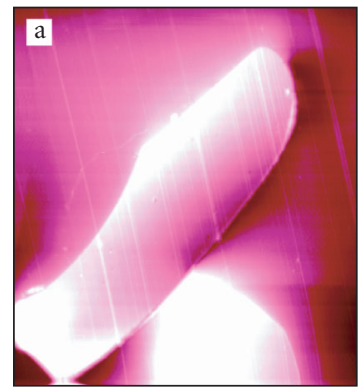

(a)

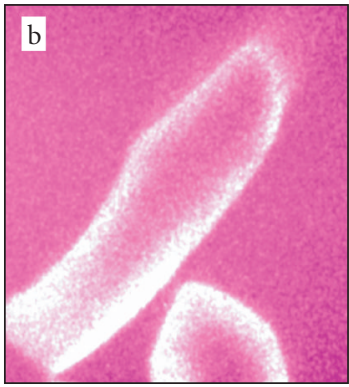

(b)

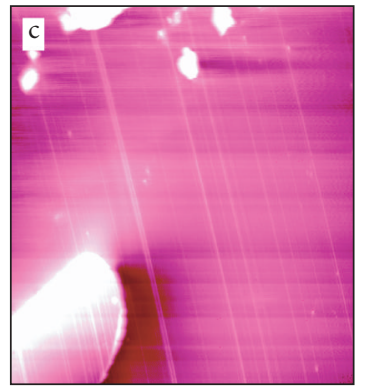

(c)

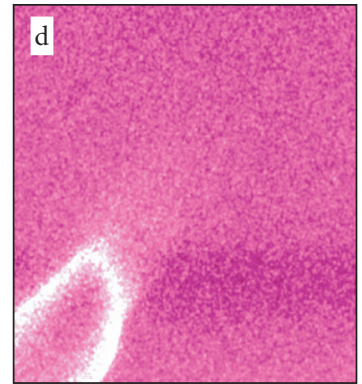

(d)

Figure 44: Simultaneous shear-force AFM (a) $(50 \mu \mathrm{m}, \mathrm{Z}$ range $1 \mu \mathrm{m})$, (c) (50 $\mu \mathrm{m}, \mathrm{Z}$ range $560 \mathrm{~nm}$ ), and fluorescence SNOM (b), (d) of microtome cut surfaces of a partly dyed polyester fiber (dye 14) embedded in Agar 100, showing the ring dyeing in the optical contrast.

The diffusion coefficient of dye $\mathbf{1 4}$ into the polyester fiber is determined with the Technovit 7100 resin microtome cut of Figure 45, which has the fiber down in order to demonstrate the versatility of the invaluable new application of artifact-free SNOM in the presence of very high topography. The right profile part is selected for the determination applying Fick's second law as above. The D-values are again constant over the complete intensity profile and the absolute value calculates to $D_{130^{\circ} \mathrm{C}}=2.4 \cdot 10^{-11} \mathrm{~cm}^{2} \mathrm{~s}^{-1}$ for the dye $\mathbf{1 4}$ into polyester fiber. This value is consistent with similar values in the literature [16]. The ease of experiment and setup guarantee widespread industrial use of this unexpected application of SNOM. Heat-transfer techniques in dyeing can be used to control the penetration depths of dyes into the fibers if the diffusion coefficients are known. Such technically important applications of SNOM are only possible with the easy and economic shear-force SNOM with apertureless (uncoated) sharp fiber tips but not with any one of the other approaches that cannot handle (high) topography.

\subsection{SNOM on fluorescing nanoparticles.}

The present SNOM technique with its lateral resolution power better than $10 \mathrm{~nm}$ is well suited for the investigation of nanoparticles such as technical dye pigments that are embedded in resin or varnish. The color shade depends strongly on their possible aggregation that can be determined by the comparison of topography width and SNOM width if the particles are covered by the embedding material. Degree and type of aggregation can be studied at the surface if part of the particles swim up in the hardening resin/varnish and contribute to the solid surface roughness. For example, fluorescent technical dye nanoparticles of 100-200 nm diameter in polyvidone resin of $1 \mu \mathrm{m}$ thickness on glass can be subjected to fluorescence SNOM with uncoated sharp tips in the shearforce gap at $488 \mathrm{~nm}$ using the edge filter OG 515 (transmitting from $506 \mathrm{~nm}$ onward; Schott, Germany) in front of the detector [16]. Figure 46 shows the topography (Figure 46(a)) on the surface and the local fluorescence (Figure 46(b)) at the 


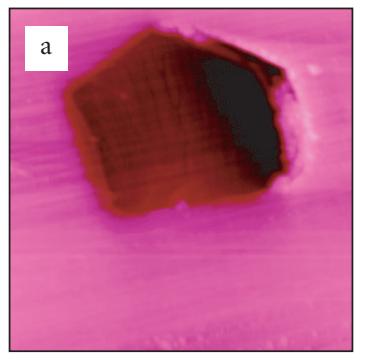

(a)

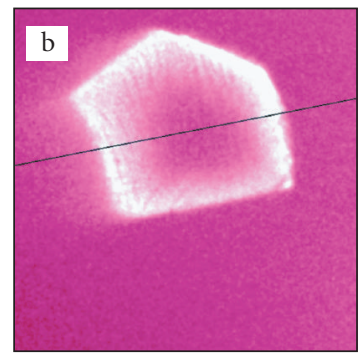

(b)

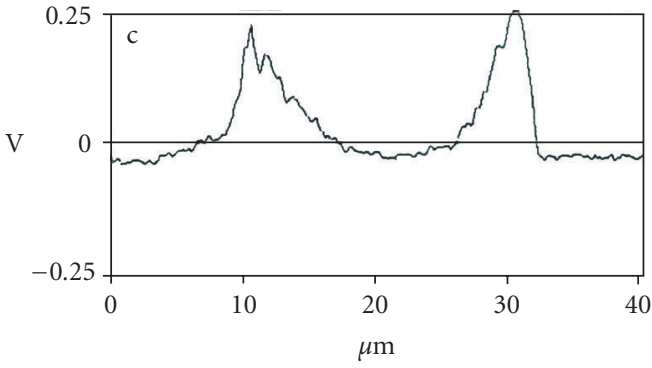

(c)

FIGURE 45: Simultaneous shear-force AFM (a) $(40 \mu \mathrm{m}, \mathrm{Z}$ range $2 \mu \mathrm{m})$ and fluorescence SNOM (b) of a microtome cut surface of a dyed polyester fiber (dye 14) embedded in Technovit 7100, showing ring dyeing in the optical contrast and the cross section (c) through the fluorescence intensity profile as indicated in (b).

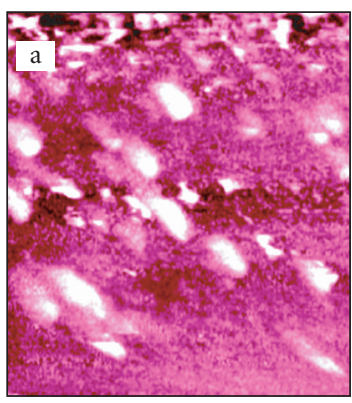

(a)

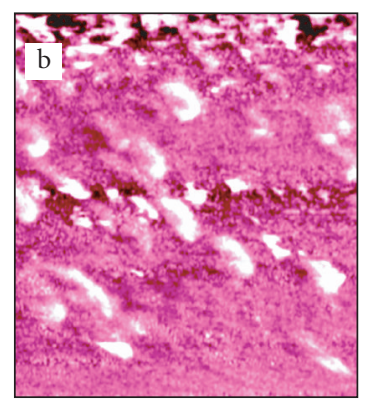

(b)
Figure 46: Simultaneous apertureless shear-force AFM (a) $(5 \mu \mathrm{m}$, $\mathrm{Z}$ range $200 \mathrm{~nm}$ ) and fluorescence SNOM (b) of nanoparticles in polyvidone resin showing the occurrence of both single and aggregated particles.

sites of the protrusions. The sizes of the protrusions indicate both single particles and aggregated ones. They emerge over the plain surface by up to $69 \mathrm{~nm}\left(\mathrm{R}_{\mathrm{ms}}=15.3 \mathrm{~nm}\right)$. The single particles have half widths of 110-210 nm in the AFM and consistently 100-200 nm in the SNOM image. Only the optical image gives the correct size of the particles. It is thus clear that the polyvidone cover on the surface particles is about $5 \mathrm{~nm}$ wide. This important result underlines the high resolving power of our SNOM. Due to the resin cover, the nanoscopic fluorescence widths are indeed smaller than the topographic features.

Furthermore, there are some remarkable differences between topographical and optical image in the larger features that correspond to aggregates. Three types of aggregation occur: linear, angular, and dense. In particular the angular ones require scrutiny at higher magnification (Figure 47). It is clearly seen in the fluorescence images (Figures 47(b), ( $\left.b^{\prime}\right)$ ) that the aggregation is angular (and there is also a single particle). As these nanoparticles form a loop, the resin cover fills the void for closing the topographic object. Only SNOM differentiates between the different chemical species. Such in- formation is of major importance for the technical application in dyed varnish formulations (surface quality and color shade). It can only be obtained with the most reliable apertureless shear-force SNOM with sharp tips under the conditions of enhancement in the shear-force gap $[1,4-7,10,15]$.

\section{CONCLUSIONS}

It could be shown that shear-force apertureless SNOM, which relies on our new photophysical effect of the strong enhancement of reflectivity in the shear-force gap under a sharp tip, is able to scan very rough "real world" samples in very different branches of the sciences without topographical artifacts. If the topography is impossible for scanning techniques (steepness close to vertical and overhangs), there is easily traced coexistence of steepness artifacts and true chemical contrast in the same image. At normal topographies (steepness up to $70-80^{\circ}$ ) artifacts occur only if tips are blunt or broken, but there are easy means to assess valid conditions for reliable measurements. The wealth of this new photophysical effect, which provides for chemical contrast, has been experienced with numerous chemical, biological, medicinal, and industrial applications. These include detailed elucidations of mechanisms in new and sustainable solid-state chemistry that were not at all thinkable on the basis of the previous topochemistry hypothesis in textbooks. Rather molecules must migrate far distances within the crystal along cleavage planes or channels or to voids from the beginning, in order to release extremely high local pressure if they change their shape upon chemical reaction [7, 11, 22, 23]. Questions of petrification of prehistoric organic fossils are successfully tackled by diffraction of the reflected light for local RAMAN SNOM as collected in the shear-force gap. Biological cell organelles are identified and optically resolved without staining, which opens new approaches to histological research at the nanoscale also for medicine with very promising applications in precancer diagnosis. Industrial applications of SNOM for "real world" surfaces profit from detection of nanoscopic pitting and immediate withdrawal of a widely used dental alloy with substitution by pitting-free materials, from nanoscopic optical analysis of paper glazing, from 


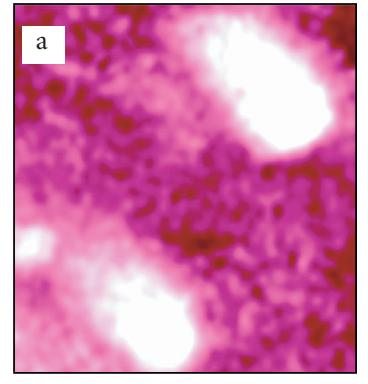

(a)

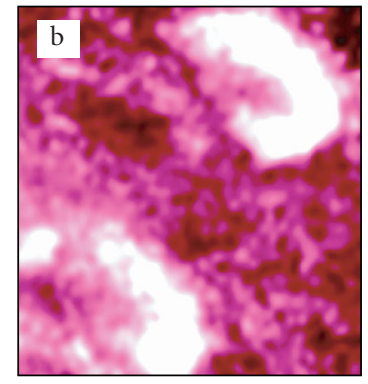

(b)

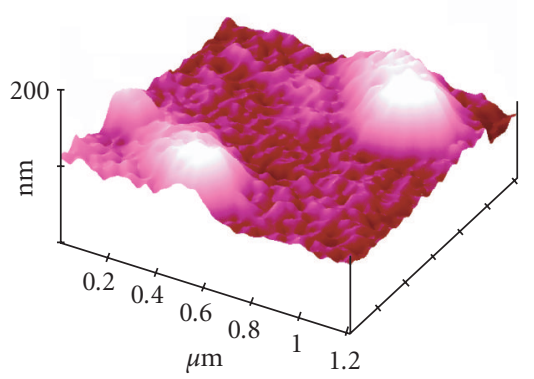

$\left(a^{\prime}\right)$

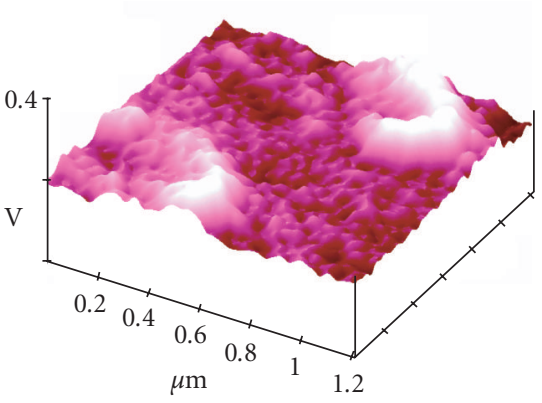

$\left(b^{\prime}\right)$

FIGURE 47: Simultaneous apertureless shear-force AFM (a), ( $\left.a^{\prime}\right)$ and fluorescence SNOM (b), ( $\left.b^{\prime}\right)$ of dye nanoparticles in polyvidone resin showing angular aggregation by the difference in shape of topographic and optical image both in the top views (a), (b) and in the surface views $\left(a^{\prime}\right),\left(b^{\prime}\right)$.

substantiation of blood bag storage lifetime with SNOM, from quality assessment of colloidal SERS materials with SNOM, and from new practice in dyeing technologies by heat transfer or nanoparticle embedding in resins/varnishes. The latter applications use apertureless fluorescence SNOM in the shear-force gap and are able to easily determine diffusion coefficients, light fastness directly in the textile fiber, aggregation types of nanoparticles, and width of the resin/varnish covering at the surface.

In none of the very diverse applications is surface roughness troublesome. The usual crosstalk that generates topographical (and further) artifacts in other SNOM techniques is absent here due to the totally independent enhancement of reflection in the shear-force gap with very sharp uncoated tips. This enhancement depends on the chemical composition and thus gives rise to chemical contrast that governs the optical image. Only apertureless SNOM in constant shearforce damping ( $\approx$ distance) with reflection back to the very sharp and easily pulled tapered waveguide on rough surfaces illuminates and collects reflected light in the extreme nearfield, the gap being only about $5 \mathrm{~nm}$. Fortunately, this wellestablished technique is particularly easy, reliable, cheap, and versatile (oblique and transparent rough or flat samples), unlike the highly complicated further SNOM approaches with their major disadvantage of not being suitable for rough "real world" samples. Therefore, enhanced reflection back to the sharp tip in the shear-force gap revolutionizes the photophysical tool SNOM, which enjoys a great future.

\section{ACKNOWLEDGMENTS}

First of all I thank my coworkers, who are named in the cited publications, for their skilled enthusiasm. This work was supported by the German Ministry of Education and Research (BMBF) under the project numbers 13N7640/3 and $13 \mathrm{~N} 7519$. We thank the named project partners for the cooperation and for providing samples.

\section{REFERENCES}

[1] G. Kaupp, J. Schmeyers, M. R. Naimi-Jamal, and V. Stepanenko, "Optische Nahfeldmikroskopie auf rauhen Oberflächen mit Glasfaserspitzen in Kreuzpolarisation, Forschungsbericht des Bundesministeriums für Bildung und Forschung (BMBF)," FKZ 13N7640/3, 25.3.2003, pp. 1-22.

[2] U. C. Fischer, "Scanning near field optical microscopy," in Scanning Probe Microscopy, R. Wiesendanger, Ed., pp. 161210, Springer, Berlin, Germany, 1998.

[3] S. Patanè, P. G. Gucciardi, M. Labardi, and M. Allegroni, "Apertureless near-field optical microscopy," Rivista del Nuovo Cimento, vol. 27, no. 1, pp. 1-46, 2004.

[4] G. Kaupp, A. Herrmann, and M. Haak, "Near-field optical microscopy with uncoated tips: calibration, chemical contrast on organic crystals, and photolithography," Journal of Vacuum Science \& Technology B (Microelectronics and Nanometer Structures), vol. 15, no. 4, pp. 1521-1526, 1997.

[5] G. Kaupp and A. Herrmann, "Scanning near-field optical microscopy by near-field reflectance enhancement: a versatile 
and valid technique," Journal of Physical Organic Chemistry, vol. 12, no. 2, pp. 141-143, 1998.

[6] G. Kaupp, A. Herrmann, and M. Haak, "Artifacts in scanning near-field optical microscopy (SNOM) due to deficient tips," Journal of Physical Organic Chemistry, vol. 12, no. 11, pp. 797807, 1999.

[7] G. Kaupp, AFM, SNOM and Nanoscratching/Nanoindentation on Natural Surfaces: New Advents for the Sciences, Springer, Berlin, Germany, 2005, in press.

[8] G. Kaupp, "Organic solid-state reactions with 100\% yield," Topics in Current Chemistry, vol. 254, pp. 95-183, 2005.

[9] G. M. J. Schmidt, "Photodimerization in the solid state," Pure and Applied Chemistry, vol. 27, pp. 647-678, 1971.

[10] G. Kaupp and A. Herrmann, "Chemical contrast in scanning near-field optical microscopy," Journal of Physical Organic Chemistry, vol. 10, no. 9, pp. 675-679, 1997.

[11] G. Kaupp, "Supermicroscopy in supramolecular chemistry: AFM, SNOM and SXM," in Comprehensive Supramolecular Chemistry, J. E. D. Davies, Ed., vol. 8, pp. 381-423, Elsevier, Oxford, UK, 1996, 21 color plates.

[12] G. Kaupp and A. Herrmann, "Abfallfreie Gas/FestkörperDiazotierung mit Stickstoffdioxid und quantitative Triazensynthese ohne Flüssigphase," Journal für Praktische Chemiel Chemiker-Zeitung, vol. 339, no. 1, pp. 256-260, 1997.

[13] G. Kaupp, A. Herrmann, and J. Schmeyers, "Waste-free chemistry of diazonium salts and benign separation of coupling products in solid salt reactions," Chemistry - A European Journal, vol. 8, no. 6, pp. 1395-1406, 2002.

[14] G. Kaupp and M. Haak, "Phase rebuilding of $\alpha$-cinnamic acid in tail irradiations," Molecular Crystals \& Liquid Crystals, vol. 313, pp. 193-198, 1998.

[15] G. Kaupp, A. Herrmann, J. Schmeyers, and J. Boy, "SNOM: a new photophysical tool," Journal of Photochemistry and Photobiology A: Chemistry, vol. 139, no. 2-3, pp. 93-96, 2001, G. Kaupp, A. Herrmann, and M. Haak, SNOM (scanning near-field optical microscopy), a genuine photochemical technique, Internet Photochem. Photobiol 1998 http://www.photobiology.com/ IUPAC98/page2.htm.

[16] G. Kaupp, A. Herrmann, and G. Wagenblast, "Scanning nearfield optical microscopy (SNOM) with uncoated tips: applications in fluorescence techniques and Raman spectroscopy," in Scanning and Force Microscopies for Biomedical Applications, vol. 3607 of Proceedings of SPIE, pp. 16-25, San Jose, Calif, USA, January 1999.

[17] E. Teixeira-Neto, G. Kaupp, and F. Galembeck, "Latex particle heterogeneity and clustering in films," The Journal of Physical Chemistry B, vol. 107, no. 51, pp. 14255-14260, 2003.

[18] E. Teixeira-Neto, G. Kaupp, and F. Galembeck, "Spatial distribution of serum solutes on dry latex sub-monolayers determined by SEPM, SNOM, SC microscopy," Colloids and Surfaces A: Physicochemical and Engineering Aspects, vol. 243, no. 1-3, pp. 79-87, 2004.

[19] G. Kaupp, A. Herrmann, M. R. Naimi-Jamal, S. Stepanenko, and V. Stepanenko, "Nahfeldmikroskopie für organische und biologische Oberflächen, Forschungsbericht des Bundesministeriums für Bildung und Forschung (BMBF)," FKZ 13N7519, 30.6.2003, pp. 1-33.

[20] W. Hevers and H. Stieve, "Ultrastructural changes of the microvillar cytoskeleton in the photoreceptor of crayfish Orconectes limosus related to different adaptation conditions," Tissue \& Cell, vol. 27, no. 4, pp. 405-419, 1995.

[21] L. F. Garfias and D. J. Siconolfi, "In-situ high-resolution microscopy on duplex stainless steels," Proceedings_Electrochemical Society, vol. 99-28, p. 89, 2000.
[22] G. Kaupp, "Solid-state molecular syntheses: complete reactions without auxiliaries based on the new solid-state mechanism," CrystEngComm, vol. 5, pp. 117-133, 2003.

[23] G. Kaupp, "Solid-state reactions, dynamics in molecular crystals," Current Opinion in Solid State and Materials Science, vol. 6, pp. 131-138, 2002. 


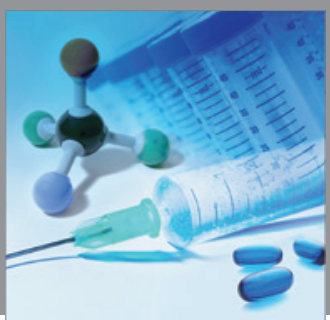

International Journal of

Medicinal Chemistry

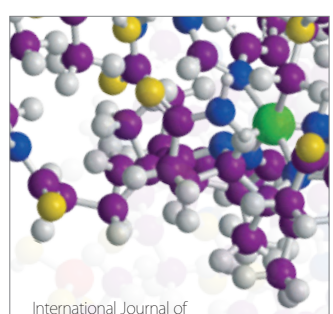

Carbohydrate Chemistry

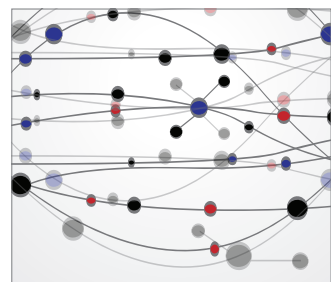

The Scientific World Journal
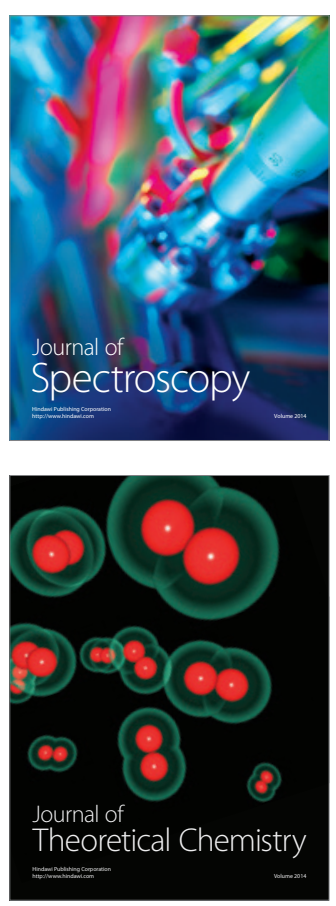
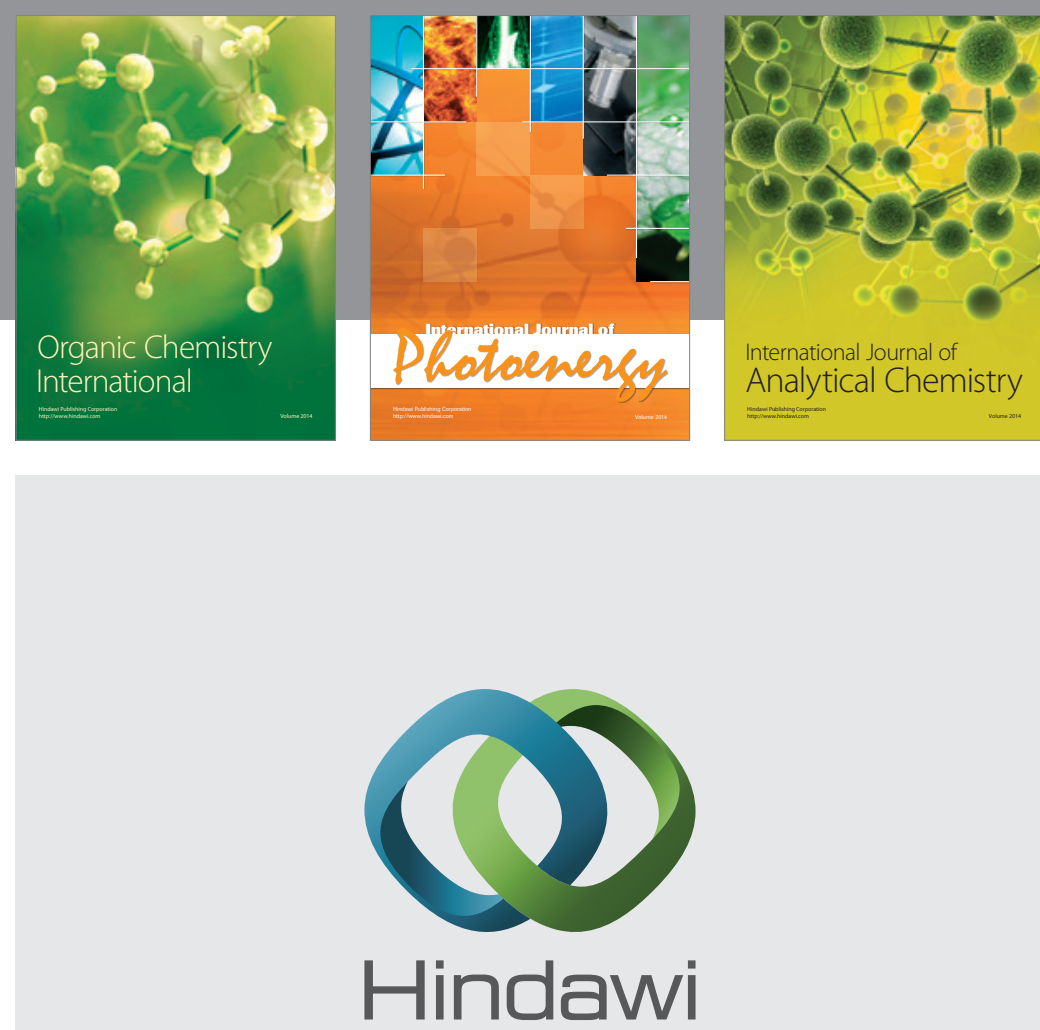

Submit your manuscripts at

http://www.hindawi.com
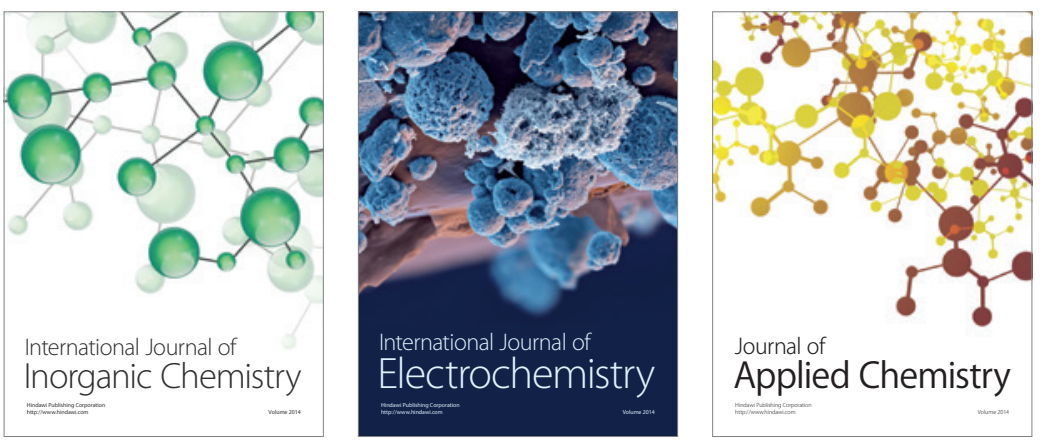

Journal of

Applied Chemistry
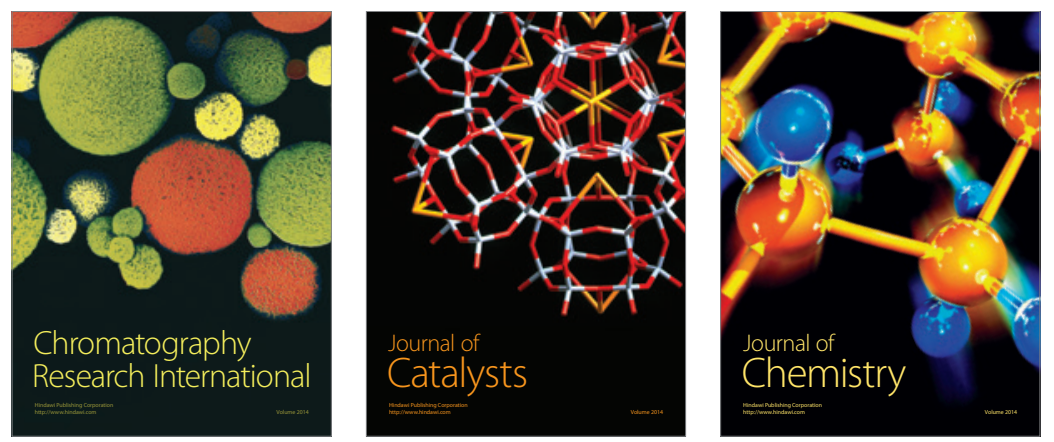
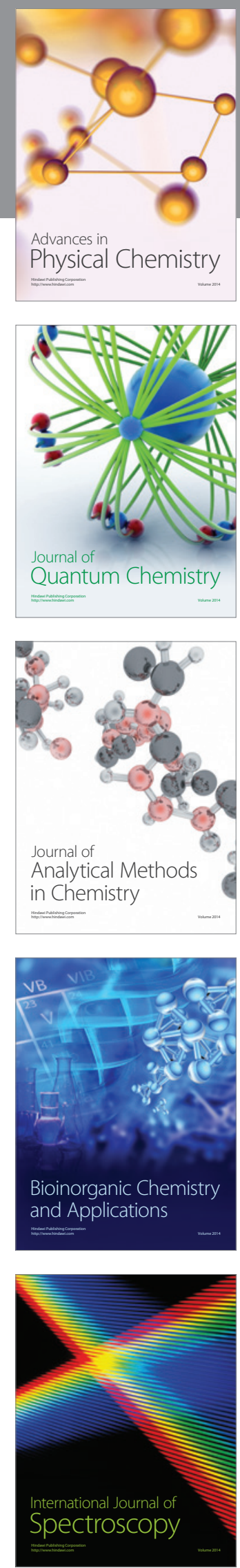